\title{
Scaling Regimes, Crossovers, and Lattice Corrections in 2D Heisenberg Antiferromagnets
}

\author{
Norbert Elstner*, Rodney L. Glenister and Rajiv R.P. Singh \\ Department of Physics, University of California, Davis, CA 95616 \\ Alexander Sokol \\ Department of Physics and Frederick Seitz Materials Research Laboratory, \\ University of Illinois at Urbana-Champaign, Urbana, IL 61801 \\ and L.D. Landau Institute, Moscow, Russia
}

(October 1, 2018)

\begin{abstract}
We study scaling behavior in $2 \mathrm{D}$, square lattice, $S=1 / 2$ and $S=1$ Heisenberg antiferromagnets using the data on full q-dependences of the equal time structure factor and the static susceptibility, calculated through high temperature series expansions. We also carry out comparisons with a model of two coupled $\mathrm{S}=1 / 2$ Heisenberg planes with the interlayer exchange coupling tuned to the $T=0$ critical point (two-plane model hereafter). For both $\mathrm{S}=1 / 2$ and $\mathrm{S}=1$ models, we separately determine the spin-wave velocity $c$ and mass $m=c / \xi$, in addition to the correlation length, $\xi$, and find that $c$ is temperature dependent; only for temperatures below $T \lesssim J S$, where $J$ is the exchange coupling, $c$ approaches its known $T=0$ value, $c_{0}$. This non-universal lattice effect is caused by the quantum nature of spin, and is therefore not captured by the quantum nonlinear $\sigma$-model. Despite this temperature dependence of the spin-wave velocity, full $q$ - and $\omega$-dependences of the dynamical susceptibility $\chi(\mathbf{q}, \omega)$ agree with those of the universal scaling function, computable for the $\sigma$-model, for temperatures up to $T_{0} \sim 0.6 c_{0} / a$, and their further analysis leads us to the inference that below $T_{0}$ the $\mathrm{S}=1$ model is in the renormalized classical ( $\mathrm{RC}$ ) regime, the two-plane model is in the quantum critical (QC) regime in agreement with earlier work, and the $\mathrm{S}=1 / 2$ model exhibits a RC-QC crossover, centered around $\mathrm{T}=0.55 \mathrm{~J}$. In particular, for the $\mathrm{S}=1 / 2$ model above the RC-QC crossover and for the two-plane model at all temperatures where calculated, the obtained spin wave mass $m=c / \xi$ is in excellent agreement with the known universal QC prediction, $m \simeq 1.04 T$. In contrast, for the $\mathrm{S}=1 / 2$ model below the RC-QC crossover, and for the $\mathrm{S}=1$ model at all temperatures, the behavior agrees with the exact RC expression. For all three models, nonuniversal behavior occurs above $T \sim 0.6 c_{0} / a$. Our results strongly support the conjecture by Chubukov and Sachdev that the $S=1 / 2$ model is close enough to the $T=0$ critical point to exhibit QC behavior at intermediate temperatures.
\end{abstract}

*Present address: Service de Physique Théorique, CEA-Saclay, 91191 Gif-sur-Yvette Cedex, France. 
Contents

I Introduction

II Overview of Renormalized Classical versus Quantum Critical Scaling Behavior

III Universal Scaling Functions

IV Scaling Regimes

A Quantum Nonlinear $\sigma$-Model . . . . . .

B Heisenberg Models. . . . . . . . . .

V Conclusion

\section{APPENDIXES}

A Quantum Nonlinear $\sigma$-Model

$1 \mathrm{~N}=\infty$ Approximation . . . . . . . .

$1 / N$ Expansion ...........

B Correlation Length

\section{INTRODUCTION}

The problem of antiferromagnetism in two dimensions has recently attracted much attention, in part due to its relevance to high temperature superconductivity. Since the high temperature superconducting materials are derivatives, obtained by doping, of parent insulating layered $S=1 / 2$ antiferromagnets, theory of the insulating phase is important for understanding the unusual magnetic and transport properties in the normal state of doped materials. Our results are directly relevant to the following two materials: $\mathrm{La}_{2} \mathrm{CuO}_{4}$, which is the insulating parent compound of the $\mathrm{La}_{2-\mathrm{x}} \mathrm{Sr}_{\mathrm{x}} \mathrm{CuO}_{4}$ and $\mathrm{La}_{2-\mathrm{x}} \mathrm{Ba}_{\mathrm{x}} \mathrm{CuO}_{4}$ superconductor families, and $\mathrm{Sr}_{2} \mathrm{CuO}_{2} \mathrm{Cl}_{2}$, which is unrelated to any superconducting family, but has well studied magnetic properties, similar to those of $\mathrm{La}_{2} \mathrm{CuO}_{4}$. Both materials experience antiferromagnetic transitions to the Neel state with nonzero staggered magnetization, $\mathrm{La}_{2} \mathrm{CuO}_{4}$ at $T_{N} \simeq 300 \mathrm{~K}$ and $\mathrm{Sr}_{2} \mathrm{CuO}_{2} \mathrm{Cl}_{2}$ at $T_{N} \simeq 256 \mathrm{~K}$. According to the Hohenberg-Mermin-Wagner theorem, in genuine two dimensional antiferromagnets the long range order at finite temperatures is prevented by thermal fluctuations. In real materials, small interlayer exchange interaction allows the transition to occur at a finite temperature $T_{N}$. In what follows we discuss only the properties well above $T_{N}$, where the magnetic subsystem can be considered two-dimensional.

It is well established [1] that the magnetic properties of $\mathrm{La}_{2} \mathrm{CuO}_{4}$ and $\mathrm{Sr}_{2} \mathrm{CuO}_{2} \mathrm{Cl}_{2}$ are described by the $S=1 / 2$ Heisenberg model on the two-dimensional square lattice. The Heisenberg model is defined by the following Hamiltonian:

$$
H=J \sum_{\langle i j\rangle} \mathbf{S}_{i} \mathbf{S}_{j},
$$

where $\mathbf{S}_{i}$ are local spin operators and the notation $\sum_{\langle i j\rangle}$ means that the sum is taken over all pairs of nearest neighbors. In what follows we set $k_{B}=\hbar=a=1$, where $a$ is the lattice constant, although in certain expressions we retain $a$ for clarity. The dynamical spin susceptibility $\chi(\mathbf{q}, \omega)=\chi^{\prime}+i \chi^{\prime \prime}$, is defined such that the magnetization induced by a wavevector and frequency dependent magnetic field is:

$$
\mathbf{M}(\mathbf{q}, \omega)=\chi(\mathbf{q}, \omega) \mathbf{H}_{\mathrm{ext}}(\mathbf{q}, \omega) .
$$

We calculate high temperature series expansions in powers of $\beta=J / T$ for two quantities: the static q-dependent susceptibility, defined as a response to a static, but generally nonuniform, magnetic field,

$$
\chi(\mathbf{q}) \equiv \chi(\mathbf{q}, \omega=0),
$$

and the Fourier transform of the equal time correlation function of two spin operators,

$$
S(\mathbf{q})=\sum_{r}\left\langle\mathrm{~S}_{z}(\mathbf{0}) \mathrm{S}_{z}(\mathbf{r})\right\rangle \exp (i \mathbf{q r}),
$$

where angular brackets represent thermal averages. These quantities can be expressed in terms of $\chi^{\prime \prime}(\mathbf{q}, \omega)$, which plays the role of the spectral weight for spin fluctuations, according to:

$$
\begin{array}{r}
S(\mathbf{q})=T \sum_{n=-\infty}^{+\infty} \chi\left(\mathbf{q}, i \omega_{n}\right)=\frac{1}{2 \pi} \int_{-\infty}^{\infty} d \omega \frac{\chi^{\prime \prime}(\mathbf{q}, \omega)}{\operatorname{th}(\omega / 2 T)}, \\
\chi(\mathbf{q})=\chi\left(\mathbf{q}, i \omega_{n}=0\right)=\frac{1}{\pi} \int_{-\infty}^{\infty} d \omega \frac{\chi^{\prime \prime}(\mathbf{q}, \omega)}{\omega}
\end{array}
$$

where $\omega_{n}=2 \pi n T$ is the imaginary bosonic Matsubara frequency.

Total spin conservation in the model leads to the relation

$$
\chi_{0}=g^{2} \mu_{B}^{2} \chi(\mathbf{q}=0)=g^{2} \mu_{B}^{2} T^{-1} S(\mathbf{q}=0),
$$

where $\chi_{0}$ is the bulk magnetic susceptibility, $g$ the electron gyromagnetic ratio, and $\mu_{B}$ the Bohr magneton.

We study both the case of $S=1 / 2$, which corresponds to $\mathrm{La}_{2} \mathrm{CuO}_{4}$ and $\mathrm{Sr}_{2} \mathrm{CuO}_{2} \mathrm{Cl}_{2}$, and the case of $S=1$. We also study a model of two $\mathrm{S}=1 / 2$ coupled Heisenberg planes at its critical point, $J_{2} / J_{1} \simeq 2.51 \pm 0.02$ [2]. For both $\chi(\mathbf{q})$ and $S(\mathbf{q})$, we are able to generate series for an arbitrary wavevector $\mathbf{q}$ complete to order $\beta^{14}$ for the $S=1 / 2$ model, and to $\beta^{10}$ for the $S=1$ model. For the two-plane model we set $J_{2} / J_{1}=2.5$, and generate series for the structure factor and the susceptibility at the antiferromagnetic ordering vector to order $\beta^{10}$. We use Pade approximation techniques for series analysis. We emphasize that the series converges extremely well in the 
temperature region presented in the paper. In fact, with the available long series, most of the Pade approximants are within a per cent, or so, from each other, down to $T \approx 0.4 J$ for $S=1 / 2$ and $T \approx 1.5 J$ for $S=1$. This allows us to put quite small errorbars on our results for all but the lowest temperatures. At the lowest temperatures, the approximants begin to significantly differ from each other and hence we do not go to still lower temperatures.

\section{OVERVIEW OF RENORMALIZED CLASSICAL VERSUS QUANTUM CRITICAL SCALING BEHAVIOR}

Scaling behavior of the 2D Heisenberg models follows from their mapping [3] to the quantum nonlinear sigma model ( $\sigma$-model hereafter). When the long range order is present at $T=0$, fluctuations at low enough temperatures are purely classical [3], in other words the characteristic frequency of spin fluctuations $\bar{\omega} \ll T$. This happens because for $T \ll \rho_{s}$ ( $\rho_{s}$ is the $\mathrm{T}=0$ spin stiffness),

$$
\bar{\omega} \sim T^{1 / 2} \exp \left(-2 \pi \rho_{s} / T\right)
$$

decreases faster than the temperature as the temperature decreases. Accordingly, this regime is called the renormalized classical ( $\mathrm{RC}$ ) regime, where "renormalized" means that the $T=0$ parameters, such as the spin stiffness, $\rho_{s}$, and spin wave velocity, $c$, are renormalized compared to their mean-field values as a result of quantum fluctuations at shorter wavelengths.

Recently, an asymptotic expression for the correlation length has been derived for this case including the exact value of the prefactor [4]:

$$
\xi=\frac{e}{8} \frac{c}{2 \pi \rho_{s}} \exp \left(\frac{2 \pi \rho_{s}}{T}\right)\left(1-\frac{T}{4 \pi \rho_{s}}+O\left[\frac{T^{2}}{\rho_{s}^{2}}\right]\right),
$$

where $e$ is the natural logarithm base. Here $\xi$ is defined such that the dominant behavior of the pair correlation function at large distances is $\exp (-r / \xi)$ with power-law corrections.

The quantum critical (QC) regime is the high temperature scaling regime in the phase diagram of the $\sigma$-model, derived by Chakravarty, Halperin and Nelson [3], where the dominant energy scale is set by the temperature:

$$
\bar{\omega} \sim T
$$

The crossover between $\mathrm{RC}$ and $\mathrm{QC}$ regimes occurs at $T \sim$ const $\times \rho_{s}$.

As the temperature increases even further, the assumptions behind the mapping of the Heisenberg model to the $\sigma$-model eventually become invalid, which at temperatures $T \gtrsim c / a$ would destroy scaling altogether.

Chubukov and Sachdev [5] recently argued that crossover from $\mathrm{RC}$ to $\mathrm{QC}$ regime occurs around $T=0.4 \mathrm{~J}$ for the $\mathrm{S}=1 / 2$ model. According to their paper, in the region of $T=0.4 J-0.6 J$, the system exhibits QC behavior. The conclusions in [5] were based on the agreement of quantum critical scaling predictions for the bulk susceptibility, $\chi_{0}$, and the NMR spin-lattice relaxation rate, $1 / T_{1}$, with the experimental measurements [6, in $\mathrm{La}_{2} \mathrm{CuO}_{4}$ and with numerical calculations for the Heisenberg model.

However, the temperature dependence of the correlation length did not appear to support this picture of the QC behavior at intermediate temperatures [8]. $\xi(T)$ has been measured by means of inelastic neutron scattering in both $\mathrm{La}_{2} \mathrm{CuO}_{4}$ [9] and $\mathrm{Sr}_{2} \mathrm{CuO}_{2} \mathrm{Cl}_{2}$ [8], and calculated numerically for the 2D Heisenberg model using Monte-Carlo simulations [10] and series expansions approaches 11. All experimental measurements and numerical results agree well with each other, and the $\mathrm{RC}$ expression seems to describe well the combined experimental and numerical data for the correlation length not only for $T \lesssim \rho_{s}$ [3], but even for temperatures as high as $T=J \sim 5 \rho_{s}$ [8]. At $T=J$, the second term in the expansion in powers of $T / \rho_{s}$ is only about two times smaller than the first term, therefore Eq.(8) may remain accurate to all orders only if the coefficients in front of higher terms in the series in $T / \rho_{s}$ are anomalously small. Since these coefficients are presently unknown and may indeed turn out to be small, on the basis of the correlation length data alone one would be tempted to conclude that the system remains in the $\mathrm{RC}$ regime for temperatures up to $T=J$, which would also imply an absence of a $\mathrm{QC}$ regime for this model [8].

The main thesis of the present paper is that such a conclusion is not justified if one examines the detailed wave-vector and temperature dependences of $S(\mathbf{q})$ and $\chi(\mathbf{q})$. It has been shown in our earlier paper [12] that the scaling functions for the static susceptibility $\chi(\mathbf{q})$, and equal time correlation function, $S(\mathbf{q})$, in the temperature range $T=0.6 J-J$ are that of the $\sigma$-model in the QC rather than the $\mathrm{RC}$ regime. In that temperature range, we also obtained various universal dimensionless ratios in excellent agreement with their respective QC predictions, with the RC predictions being far outside the errorbars.

However, the temperature dependence of the correlation length still presented a puzzle. In the quantum critical region, the leading temperature dependence of the correlation length is of the form

$$
\xi=X_{\infty}^{-1} \frac{c}{T}
$$

where $c$ is the spin-wave velocity and $X_{\infty}$ is a universal constant. With the known value of $X_{\infty}$ [3,5, 13, and the known zero temperature value of the spin-wave velocity, the temperature dependence of the correlation length did not fit Eq.(10) in any extended temperature region, although the magnitude of $\xi$ around $T \sim J / 2$ is in agreement with Eq.(10). In Ref. [12], we conjectured that there are nonuniversal lattice corrections at intermediate temperatures, which affect such quantities as the correlation length, but nevertheless the scaling functions and 
the characteristic frequency of spin fluctuations are that of the QC regime.

In the present paper, we are able to show that the disagreement of the correlation length with the quantum critical prediction is indeed a result of lattice corrections, more specifically corrections to the spin wave velocity, which is temperature dependent especially at higher temperatures. We further show that when Eq.(10) is evaluated using the correct $c(T)$ rather than its $T=0$ value, the agreement with the QC behavior is observed for temperatures above $T \sim 0.55 \mathrm{~J}$.

\section{UNIVERSAL SCALING FUNCTIONS}

The first stage of our analysis is to establish whether or not, and in what temperature range, universal $q-$ and $\omega$-dependences of $\chi(\mathbf{q}, \omega)$, derived for the quantum nonlinear $\sigma$-model, apply to the $\mathrm{S}=1 / 2$ and $\mathrm{S}=1$ square lattice Heisenberg antiferromagnets. Here, it is convenient to introduce the spin wave mass defined as

$$
m(T)=\frac{c(T)}{\xi(T)},
$$

which, in what follows, replaces $\xi(T)$ as a scaling parameter. We emphasize that both $c(T)$ and $\xi(T)$ are measurable thermodynamic quantities. This replacement facilitates scaling comparisons: we will show that when at temperatures $T \sim J S$ the spin wave velocity becomes temperature dependent due to lattice corrections to $c$, the behavior of $m$ remains universal.

For the $\sigma$-model calculations, we employ $1 / N$ expansion approach and utilize some of the results obtained earlier by Chubukov and Sachdev [5]. We do not calculate the frequency dependence of the dynamical susceptibility explicitly. Instead, we base our comparisons on the calculated difference between the equal time correlators and static susceptibilities at all wavevectors, which is a sensitive probe of the frequency distribution of the spectral weight. Our calculations are discussed in detail in Appendix A, where we derive the following equations which relate $S(\mathbf{q})$ and $\chi(\mathbf{q})$, computable via series expansions for the Heisenberg models, to the scaling parameters $m$ and $c$ :

$$
\begin{gathered}
\frac{\chi(\mathbf{Q})}{\chi(\mathbf{q})}=1+\left(\frac{c}{m}\right)^{2}|\mathbf{q}-\mathbf{Q}|^{2}, \\
\gamma^{2}\left[\frac{S(\mathbf{q})}{T \chi(\mathbf{q})}\right]=\left(u_{2} \frac{m}{T}\right)^{2}+\left(\frac{c}{T}\right)^{2}|\mathbf{q}-\mathbf{Q}|^{2}
\end{gathered}
$$

where

$$
u_{2} \simeq 1+0.1473 / N=1.0491
$$

and the function $\gamma[x]$ is defined according to:

$$
\gamma\left[\frac{x / 2}{\tanh (x / 2)}\right] \equiv x, \quad \text { for } \quad x>1 .
$$

Here the expression for $u_{2}$ is evaluated at $N=3$, which corresponds to the physical three-component spin. As discussed in Appendix A, these equations are not exact. In particular, there are nonzero higher order terms in $|\mathbf{q}-\mathbf{Q}|^{2}$ and multiplicative factors in front of $c$. However, such corrections are found to be less than $1-2 \%$, which greatly simplifies further considerations.

Eqs.(12,13) are in a form that greatly facilitates comparison with the numerical data: the left hand parts of the above equations are computable numerically for the Heisenberg models, and their linear dependence on $|\mathbf{q}-\mathbf{Q}|^{2}$, expected from Eqs. (12,13), can be verified by plotting on the appropriate scale. In the temperature range where the expected linear behavior is indeed observed, the scaling parameters $m$ and $c$ can be determined independently from the intercept and slope of the plotted data.

Before we proceed with the comparisons, an important remark is in order. Since at high temperatures $\chi(\mathbf{q})$ and $S(\mathbf{q})$ do not exhibit strong $q$-dependence, their small deviations from the values at $\mathbf{Q}=(\pi, \pi)$ are always linear in $|\mathbf{q}-\mathbf{Q}|^{2}$, as required by the lattice symmetry, so that at high enough temperatures $q$-dependences in Eqs.(12 13) would convey no information about scaling. The high temperature crossover to non-universal weakly interacting spins is discussed more thoroughly in the next section.

We start with Eq.(12) and plot its left hand side, calculated using high temperature series expansions, versus $|\mathbf{q}-\mathbf{Q}|^{2}$ for the $\mathrm{S}=1 / 2$ and $\mathrm{S}=1$ Heisenberg models in Fig.11. Eq.(12) predicts that the plotted data should be linear. For wavevectors $|\mathbf{q}-\mathbf{Q}| \leq 0.8$, this is indeed the case for both $\mathrm{S}=1 / 2$ and $\mathrm{S}=1$ models in a broad range of temperatures: $T=0.4 J-J$ for $\mathrm{S}=1 / 2$, and $T=1.5 \mathrm{~J}-1.9 \mathrm{~J}$ for $\mathrm{S}=1$.

We now turn to Eq.(13) and plot its left hand side versus $|\mathbf{q}-\mathbf{Q}|^{2}$ in Fig.2. Again, as expected from Eq.(13), the plotted data is linear in the same wavevector and temperature range for both $S=1 / 2$ and $S=1$ models.

Both Figs.1.2, 2 are generated with $\mathbf{q}-\mathbf{Q}$ pointing along the diagonal of the Brillouin zone. The data with $\mathbf{q}-\mathbf{Q}$ pointing along other directions is virtually identical in the same wavevector and temperature range, as expected in the isotropic $\sigma$-model. Further, a straightforward estimate shows that corrections to linear spin wave spectrum due to higher gradients, not included in the $\sigma$-model, are only about $5 \%$ for the wavevector range of interest, $|\mathbf{q}-\mathbf{Q}|<0.8$. The studied temperature range is limited from below by our ability to evaluate accurately $\chi(\mathbf{q})$ and $S(\mathbf{q})$ from our high temperature series, presently known to $\beta^{14}$ for the $\mathrm{S}=1 / 2$ model, and to $\beta^{10}$ for the $\mathrm{S}=1$ model.

From the slope and intercept of the linear fits to the data plotted in Fig.2, we are able to determine $c$ and $m$ separately. Since this data is used extensively in what follows, we carry out comparisons of our results for $m$ 
and $c$ with direct calculations of the correlation length. The comparisons, which are described in Appendix B, confirm that our calculations are indeed accurate.

We have thereby established that Eqs.(12,13) are valid in the range $T=0.4 J-J(T=1.5 J-1.9 J)$ for $\mathrm{S}=1 / 2$ $(\mathrm{S}=1)$ for $|\mathbf{q}-\mathbf{Q}| \leq 0.8$, which means that wavevector dependences of $S(\mathbf{q})$ and $\chi(\mathbf{q})$ in the Heisenberg models are described by the $\sigma$-model in the same wavevector and temperature range, which is shaded in Fig. 3 .

\section{SCALING REGIMES}

\section{A. Quantum Nonlinear $\sigma$-Model}

We now proceed with the discussion of scaling regimes. The dynamical scaling form of the staggered correlator is [3]:

$$
\chi_{s}(q, \omega)=\chi_{s}(0) \Phi^{(i)}\left(\frac{c q}{m}, \frac{\omega}{\bar{\omega}}\right)
$$

where the scaling function for the renormalized classical regime, $\Phi^{\mathrm{RC}}$, differs from that in the quantum critical regime, $\Phi^{\mathrm{QC}}$. In general, $\bar{\omega}$ and $m$ are not equivalent: the spin wave velocity, and therefore $m$, are defined at $q \gg \xi^{-1}$, while $\bar{\omega}$ is defined at $q \sim \xi^{-1}$. In the quantum nonlinear $\sigma$-model, $c$ is expected to be temperature dependent only when the temperature is comparable to the ultraviolet cutoff $\Lambda$ (except in case of relativistic invariance of the cutoff procedure, when $c$ is always temperature independent and coincides with its bare value).

In the $\mathrm{RC}$ regime temperature dependences of the spin wave mass for $N=\infty$ and $N=3$ are different: [3,5]:

$$
m \sim \exp \left(-\frac{2 \pi \rho_{s}}{T}\right) \times\left\{\begin{array}{ll}
T & \text { for } N=\infty \\
\rho_{s} & \text { for } N=3
\end{array} .\right.
$$

Furthermore, in this regime $\bar{\omega}$ is not equivalent to $m$, except for $N=\infty$ :

$$
\frac{\bar{\omega}}{m} \sim \begin{cases}1 & \text { for } N=\infty \\ \left(T / \rho_{s}\right)^{1 / 2} & \text { for } N=3\end{cases}
$$

The exact asymptotic expression for $m$ follows immediately from Eq.(8):

$$
m=\frac{16 \pi \rho_{s}}{e} \exp \left(\frac{-2 \pi \rho_{s}}{T}\right)\left(1-\frac{T}{4 \pi \rho_{s}}+O\left[\frac{T^{2}}{\rho_{s}^{2}}\right]\right)^{-1} .
$$

The value of $\rho_{s}$, which is the only dimensional parameter in Eq.(19), is known for the Heisenberg models from zero temperature series expansions [14, quantum Monte Carlo simulations [15], and $1 / S$ calculations [16].

In the $\mathrm{QC}$ regime, temperature is the only energy scale in the problem, so that:

$$
\bar{\omega} \sim m \sim T
$$

which is valid for both $N=3$ and $N=\infty$ models. Furthermore, the ratio $m / T$ is universal in the $\sigma$-model, and has been calculated via the $1 / N$ approach [5]:

$$
\frac{m}{T}=\Theta\left(1+\frac{0.2373}{N}\right) \sim 0.9624+\frac{0.2284}{N} \approx 1.04
$$

where $\Theta=2 \log [(1+\sqrt{5}) / 2]$. Given that already the first subleading $1 / N$ correction is small $(\sim 8 \%)$ for $N=3$, Eq.(21) is likely to be close to the true value for the $O(3) \sigma$-model, and is hereafter adopted as the QC prediction. It can be compared with calculations based on other techniques:

$$
\frac{m}{T}=\left\{\begin{array}{ll}
0.9, & 2+\epsilon \text { expansions }[3] \\
1.25 \pm .25, & \text { Monte Carlo 13 }
\end{array} .\right.
$$

The results are generally consistent with each other. Further support for the numerical value obtained in the $1 / N$ calculation comes from the study of the two-plane model at its $T=0$ critical coupling by Sandvik and Scalapino [2], where temperature dependence of the correlation length was found to be in agreement with that derived from Eq. 21).

Thus, both RC (19) and QC (21) predictions for $m(T)$ are available to carry out comparisons with Heisenberg models without any adjustable parameters.

\section{B. Heisenberg Models}

We start by comparing behavior of the spin wave mass for $\mathrm{S}=1 / 2, \mathrm{~S}=1$, and the two plane models. The $\mathrm{S}=1$ model, due to its larger spin, is "more classical" than the $\mathrm{S}=1 / 2$ model, hence it is less likely to have a range of quantum critical behavior. On the other hand, for the two plane model at its critical point, temperature dependences of $\chi_{0}$ and $\xi$ consistent with the $\mathrm{QC}$ regime had been reported earlier by Sandvik and Scalapino [2]. The comparison of different models proves quite informative.

We now plot $m(T)$ for these three models along with the universal RC (19) and QC (21) scaling predictions in Fig. 4 . It is evident from Fig. 4 that $m(T)$ for the $\mathrm{S}=1$ model shows $\mathrm{RC}$ behavior at all temperatures, for the two plane model QC behavior at all temperatures, and for the $\mathrm{S}=1 / 2$ single plane model a crossover between RC behavior below $T \sim 0.45 J$ and QC behavior above $T=0.65 J$.

We now discuss the onset of nonuniversal behavior at high temperatures. The temperature scale at which nonuniversal behavior arises is set roughly by $c / a$. The spin wave spectrum becomes nonlinear at $q \sim a^{-1}$, and the excitations in this wavevector range are suppressed only when $T \ll c / a$. The spin stiffness, on the other hand, is less relevant to this crossover to nonuniversal behavior at high temperatures. To a crude approximation we expect scaling to fail for $\mathrm{S}=1 / 2$ and $\mathrm{S}=1$ models at $T /(c / a)$ of the same order, given their similar spin wave 
spectrum. In order to study failure of scaling, we therefore plot the ratio $m / T$ versus $T /\left(c_{0} / a\right)$ (Fig.5). The use of $c_{0}$ instead of the temperature dependent $c(T)$ is to avoid a nonlinear temperature scale. In this particular case, the difference between $c$ and $c_{0}$ is less important given the ambiguous character of the upper boundary of scaling behavior.

One can see from Fig. 5 that deviations from the universal behavior of $m$ occurs at similar values of the ratio $T /\left(c_{0} / a\right) \sim 0.6$ for both $\mathrm{S}=1 / 2$ and $\mathrm{S}=1$ models, above which a crossover to the $T \gg J$ asymptotics $m \sim 1 / T$ occurs. The region where $m$ becomes nonuniversal and no longer represents the spin wave mass is shaded in Fig. 5 . Expressed in terms of $J$, the range where universal behavior of $m(T)$ holds is below $T \sim J$ for the $\mathrm{S}=1 / 2$ model and below $T \sim 1.9 J$ for the $\mathrm{S}=1$ model.

Now, we turn to the analysis of the spin wave velocity calculated in Sect IIII and plot $c$ determined from the data at different temperatures using Eq.13 in Fig.6.

Dashed lines show the known $T=0$ value of the spin wave velocity calculated using $1 / S$ expansions 16 ,

$$
\begin{array}{rl}
c=2 \sqrt{2} J & S\left(1+0.1580 / 2 S+0.0216 /(2 S)^{2}\right) \\
& \simeq \begin{cases}1.67 J & \text { for } \mathrm{S}=1 / 2 \\
3.07 J & \text { for } \mathrm{S}=1\end{cases}
\end{array}
$$

which for $\mathrm{S}=1 / 2$ compares favorably with the $\mathrm{T}=0$ series expansion calculations 14.

For all temperatures studied the spin wave velocity turns out to be temperature dependent for both $\mathrm{S}=1 / 2$ and $\mathrm{S}=1$ models. Towards the lowest temperatures in the numerically accessible range, $c$ approaches the expected $\mathrm{T}=0$ values given by Eq.(23). Since in the $\sigma$ model the spin wave velocity is temperature independent except near the ultraviolet cutoff, which in our case is set by the scale of order $c / a \sim 2 \sqrt{2} J S$, the observed temperature dependent spin wave velocity clearly results from the quantum nature of spin, when the temperature becomes comparable to $J S$.

The quantum corrections to the spin wave velocity originating from higher order $1 / S$ terms in the spin wave theory, were calculated by Kaganov and Chubukov [19]:

$$
c(T)=c_{0}\left[1+\frac{\zeta(3)}{8 \pi S}\left(\frac{T}{S J}\right)^{3}\right]^{-1},
$$

where $\zeta(x)$ is the Riemann zeta function. The predictions of Eq. 24), evaluated for $\mathrm{S}=1 / 2$ and $\mathrm{S}=1$, are shown as solid lines in Fig.6a,b, respectively. The agreement with numerical calculations is quite good. Despite higher order terms in the expansion in powers of $T / J S$, it appears that Eq.24 describes the observed temperature dependence of $c$ fairly well for both $S=1 / 2$ and $S=1$.

At this stage an important question is, does the temperature dependence of $c$ destroy scaling altogether? One might expect that once lattice corrections become important for quantities like the spin-wave velocity, they might also lead to a breakdown of scaling altogether by introducing deviations from linearity in the spin wave spectrum and appearance of spectral weight in modes not contained in the quantum nonlinear $\sigma$-model. We argue that this is not the case here. Indeed, from the fact that Eqs.(12,13) remain valid when $c(T)$ is already temperature dependent, we conclude that the spin wave spectrum still remains linear even though $c(T)$ deviates from its $T=0$ value. By the same token, we conclude that there are no modes with significant spectral weight other than those described by the $\sigma$-model.

Another universal quantity which has not been discussed in our paper so far is the overall prefactor, $\chi_{Q}$, in front of the scaling expression for $\chi(\mathbf{q}, \omega)$ in Eq.(16). This prefactor, which is renormalized by short wavelength quantum fluctuations, can be expressed in terms of $N_{0}$ and $\rho_{s}$ for temperatures where corrections of order $T / J S$ can be ignored. Where $c$ is $T$-dependent, this quantity becomes $T$-dependent as well. However, quantum corrections to the prefactor due to $1 / S$ terms have not yet been calculated analytically.

Now the reason why the quantum critical scaling fails to describe the temperature dependence of the correlation length is clear. The correlation length derived from Eq.(A3) is $\xi=c / m$. If one assumes that the spin wave velocity is temperature independent above $T=0.4 J$, the quantum critical expression does not agree with the numerical data. Given the discussions above, we argue that one should consider not the temperature dependence of $\xi$, but that of $m=c / \xi$, because $m$ is unaffected by the lattice corrections to $c$. Then, the crossover from RC behavior to QC behavior at temperatures around $J / 2$ becomes evident.

\section{CONCLUSION}

Magnetic properties of the doped superconducting cuprates may be closely related to the undoped parent compounds, such as $\mathrm{La}_{2} \mathrm{CuO}_{4}$ and $\mathrm{Sr}_{2} \mathrm{CuO}_{2} \mathrm{Cl}_{2}$, which are well described by the square lattice Heisenberg model. Motivated by this relationship, we have studied scaling and the role of lattice corrections in two dimensional square lattice Heisenberg antiferromagnets.

In [3], Chakravarty, Halperin, and Nelson mapped 2D collinear quantum antiferromagnets onto the quantum nonlinear $\sigma$-model, and derived the corresponding phase diagram. When the $\mathrm{T}=0$ ground state of the corresponding $\sigma$-model has Neel order, as is the case for the square lattice Heisenberg models, the low temperature scaling regime is classical (often called renormalized classical, or $\mathrm{RC}$ ), where the characteristic energy scale $\bar{\omega} \ll T$, while at higher temperatures a crossover to the quantum critical (QC) regime may be observed, where $\bar{\omega} \sim T$. The $\mathrm{S}=1 / 2$ square lattice Heisenberg model, which describes $\mathrm{La}_{2} \mathrm{CuO}_{4}$, was shown to exhibit $\mathrm{RC}$ behavior at low temperatures [3]. As it has a substantial ordered moment, 
it was earlier believed not to have a region of quantumcritical behavior at intermediate temperatures.

More recently, Chubukov and Sachdev [5] argued that the crossover from the $\mathrm{RC}$ to nonuniversal microscopic behavior occurs through the intermediate QC regime at $0.4 J<T<0.6 J$, with universal behavior extending up to $T=0.6 J$ and nonuniversal behavior above that temperature. The importance of this at first sight minor difference concerning a fairly narrow temperature range, arises from the fact that upon doping the QC region was argued to expand rather rapidly, covering a much broader, and well accessible temperature range [5]. This scenario has important implications for our views of magnetism in the superconducting cuprate materials [5,20].

In our earlier paper [12, we showed that the scaling functions for the equal time spin correlator, $S(\mathbf{q})$, and the static susceptibility, $\chi(\mathbf{q})$, in the $\mathrm{S}=1 / 2$ Heisenberg model in the temperature range $T=0.6 J-J$ agree with those calculated for the $\sigma$-model in the $\mathrm{QC}$ regime. The same turned out to be true for the ratio $S(\mathbf{q}) / T \chi(\mathbf{q})$. Our comparisons did not involve any adjustable parameters.

In the present paper, we undertook a detailed study of these issues using the high temperature series expansions approach. The simultaneous study of $\mathrm{S}=1 / 2$ and $\mathrm{S}=1$ models allows us to contrast the two cases and highlight the parameter-free agreement of the intermediate temperature behavior for the $S=1 / 2$ case with the universal quantum critical predictions of the non-linear $\sigma$-model. For both $S=1 / 2$ and $S=1$ models, we find that the q-dependences of $S(\mathbf{q})$ and $\chi(\mathbf{q})$ separately, as well as of their ratio, agree very well up to $T \sim 0.6 c_{0} / a$ with the $\sigma$-model predictions calculated separately using $1 / N$ expansions (for $1 / N$ calculations, we utilized many of the results obtained in [5]). This allows us to calculate the temperature dependence of the spin wave mass, defined as $m=c / \xi$, for which both $\mathrm{RC}$ and $\mathrm{QC}$ predictions are known without adjustable parameters.

For $\mathrm{S}=1 / 2$, we find that $m(T)$ is in good agreement with the RC prediction for $T<0.45 J$, while for $0.65 J<$ $T<J$ the results are very close to the QC prediction and far from the RC prediction, exhibiting a crossover between these two regimes for $0.45 J<T<0.65 J$. Furthermore, we find that for $T>0.5 J$, the lattice corrections cause a temperature dependence of the spin-wave velocity $c$ and affect the overall prefactor in front of the scaling expression for $\chi(\mathbf{q}, \omega)$. The new results strongly support our earlier conjecture [12] that lattice corrections are such that full q- and $\omega$-dependences remain universal in the QC regime for temperatures up to $T \simeq 0.6 c_{0} / a \sim J$ :

$$
\chi(\mathbf{q}, \omega)=\chi_{Q} \Phi^{\mathrm{QC}}\left(\frac{c|\mathbf{q}-\mathbf{Q}|}{T}, \frac{\omega}{T}\right),
$$

where $\Phi^{\mathrm{QC}}(x, y)$ is the scaling function of the QC regime, computable using, for instance, the approach of [5]. For the $\mathrm{S}=1$ model, where quantum fluctuations are substantially less, we find that RC behavior directly crosses over to the high temperature interacting local-spin behavior without an intervening QC regime. Finally, the observed lattice corrections to $c$ for both $\mathrm{S}=1 / 2$ and $\mathrm{S}=1$ roughly agree in magnitude and temperature dependence with earlier calculations of quantum $T / J S$ effects by Kaganov and Chubukov [19].

In their recent publication, Greven and coworkers [8] pointed out the agreement of the measured and numerically calculated correlation length $\xi(T)$ with RC prediction for all temperatures below $T=J$. In our work, we present an alternative theory which is not only consistent with the same data for $\xi(T)$, but explains the qand $\omega$-dependences of spin correlators as well. We show that detailed examination of the wavevector and temperature dependence of $S(q)$ and $\chi(q)$ supports the picture of renormalized classical behavior for temperatures up to $T=0.45 J$, and a crossover to a quantum critical behavior around $T=J / 2$.

While the QC behavior for the spin wave mass occurs in our calculations in the temperature range $T>0.6 \mathrm{~J}$ currently inaccessible to experiments in $\mathrm{La}_{2} \mathrm{CuO}_{4}$ and $\mathrm{Sr}_{2} \mathrm{CuO}_{2} \mathrm{Cl}_{2}$, many signatures of the $\mathrm{QC}$ behavior have been experimentally observed at lower temperatures. For instance, the nuclear relaxation rate, $1 / T_{1}$, in $\mathrm{La}_{2} \mathrm{CuO}_{4}$ saturates above $T=600 K \sim 0.4 J$ [7 at a value close in magnitude to the universal QC prediction [5]. This is consistent with the present study, as it has been shown 15.21 that for those quantities which in the $\mathrm{RC}$ regime depend on $\xi$ through its logarithm only, such as the ratio $T_{1} T / T_{2 \mathrm{G}}$ and the bulk susceptibility, $\chi_{0}$, the RC-QC crossover should be shifted substantially towards lower temperatures, in agreement with the measurements of $\chi_{0}$ [6] and $T_{1} T / T_{2 g}$ [7] in $\mathrm{La}_{2} \mathrm{CuO}_{4}$.

It has been proposed earlier that the temperature range of quantum critical behavior rapidly expands with doping, and that the scaling behavior described by the quantum nonlinear $\sigma$-model may be observed in doped antiferromagnets, and specifically in the high temperature superconductors $5,20,12$. We hope that the study of the renormalized classical to quantum critical scaling crossover and of the origin of nonuniversal corrections to scaling in the insulator presented here, will be helpful in understanding magnetic behavior of the doped systems as well.

\section{ACKNOWLEDGMENTS}

We are grateful to R.J. Birgeneau, S. Chakravarty, A.V. Chubukov, M. Greven, A.J. Millis, D. Pines, S. Sachdev, A.W. Sandvik, and D.J. Scalapino for many useful discussions. N.E. was supported by the Deutsche Forschungsgemeinschaft. R.L.G. and R.R.P.S. were supported by the NSF Grant DMR93-18537. A.S. was supported by the NSF Grant DMR89-20538 through the Frederick Seitz Materials Research Laboratory. The computations have been performed using the IBM RISC/6000 workstation received through the Shared Uni- 
versity Research Grant from the IBM Corporation. N.E. would like to acknowledge the kind hospitality of A.P. Young and Physics Department of the University of California at Santa Cruz where part of this work was done. A.S. is grateful to the Aspen Center for Physics for hospitality during intial stages of this work.

[1] For comprehensive reviews, see S. Chakravarty, in Proceedings of High Temperature Superconductivity, edited by K.S. Bedell et al. (Addison-Wesley, CA, 1990), and E. Manousakis, Rev. Mod. Phys. 63, 1 (1991).

[2] A.W. Sandvik and D.J. Scalapino, Phys. Rev. Lett., 72, 277 (1994), and unpublished. The two plane model was discussed earlier by K. Hida, J. Phys. Soc. Japan, 61, 1013 (1992); A.J. Millis and H. Monien, Phys. Rev. Lett., 70, 2810 (1993).

[3] S. Chakravarty, B.I. Halperin, and D.R. Nelson, Phys. Rev. B 39, 2344 (1989); see also S. Chakravarty and R. Orbach, Phys. Rev. Lett. 64, 224 (1990).

[4] P. Hasenfratz and F. Niedermayer, Phys. Lett. B 268, 231 (1991); Z. Phys. B 92, 91 (1993).

[5] A.V. Chubukov and S. Sachdev, Phys. Rev. Lett. 71, 169 (1993); A.V. Chubukov, S. Sachdev, and J. Ye, Phys. Rev. B49, 11919 (1994).

[6] D.C. Johnston, Phys. Rev. Lett. 62, 957 (1989); D.C. Johnston, S.K. Sinha, A.J. Jacobson and J.M. Newsam, Physica (Amsterdam), 153-155 C, 572 (1988)

[7] T. Imai, C.P. Slichter, K. Yoshimura and K. Kosuge, Phys. Rev. Lett. 70, 1002 (1993); T. Imai, C.P. Slichter, K. Yoshimura, M.Katoh and K. Kosuge, Phys. Rev. Lett. 71, 1254 (1993).

[8] M. Greven et al., Phys. Rev. Lett. 72, 1096 (1994).

[9] B. Keimer et al., Phys. Rev. B 46, 14034 (1992).

[10] H.-Q. Ding and M.S. Makivic, Phys. Rev. Lett, 64, 1449 (1990); M.S. Makivic and H.-Q. Ding, Phys. Rev. B 43, 3662 (1990).

[11] R.R.P. Singh and R.L. Glenister, Phys. Rev. B 46, 11871 (1992).

[12] A. Sokol, R.L. Glenister and R.R.P. Singh, Phys. Rev. Lett., 72, 1549 (1994).

[13] E. Manousakis and R. Salvador, Phys. Rev. Lett., 62, 1310 (1989); Phys. Rev. B 40, 2205 (1989). Remark: in our opinion, the data for the slab thickness $N_{\tau}=2$ may not be appropriate for extracting properties of the thermodynamic limit $\left(N_{\tau}=\infty\right)$, where $m / T$ is defined. We therefore repeated the analysis of Manousakis and Salvador omitting the data for $N_{\tau}=2$, arriving to the errorbars for $m / T$, shown in Eq. 22, which are larger than the errorbars quoted in the original paper. With this larger erorbars, the result agrees with calculations in the framework of other approaches.

[14] R.R.P. Singh, Phys. Rev. B 39, 9760 (1989); R.R.P. Singh and D. Huse, Phys. Rev. B 40, 7247 (1989).

[15] U.J. Wiese and H.P. Ying, Z. Phys. B93, 147 (1994).

[16] T. Oguchi, Phys. Rev, 117, 117 (1960); S. Chakravarty and C. Castilia, Phys. Rev. B 43, 13687 (1991); J. Igarashi, Phys. Rev. B 46, 10763 (1992).

[17] A.V. Chubukov and A.W. Sandvik, unpublished.

[18] R.R.P. Singh, Phys. Rev. B47, 12337 (1993).

[19] M.I. Kaganov and A.V. Chubukov, in "Spin waves and Magnetic Excitations", part I, Eds. A.S. BorovikRomanov and S.K. Sinha, North-Holland, Amsterdam (1988).

[20] A. Sokol and D. Pines, Phys. Rev. Lett. 71, 2813 (1993).

[21] A.V. Chubukov, S. Sachdev, and A. Sokol, Phys. Rev. B 49, 9052 (1994).

[22] E.H. Fradkin, Field Theories of Condensed Matter Systems, Addison-Wesley, Redwood City, CA (1991).

[23] R.L. Glenister, N. Elstner, R.R.P. Singh, and A. Sokol, to be published.

\section{APPENDIX A: QUANTUM NONLINEAR $\sigma$-MODEL}

The mapping [3] of the low energy spectrum of the Heisenberg model to the quantum nonlinear sigma model has been extensively discussed in the literature and textbooks (e.g., see 22]). Here we briefly discuss some aspects of this mapping, and of the $1 / N$ expansion in this model, as necessary for our purposes. We refer the reader to Refs. 3,5] for a far more detailed treatment.

The action of the quantum nonlinear $\sigma$-model can be written as:

$$
\begin{array}{r}
S=-\frac{\rho_{s 0}}{2} \int d^{2} \mathbf{r} \int_{0}^{1 / T} d \tau\left[\frac{1}{c_{0}^{2}}\left(\partial_{\tau} \vec{n}\right)^{2}+(\nabla \vec{n})^{2}\right] \\
\vec{n}^{2}=1
\end{array}
$$

where the constraint $\vec{n}^{2}=1$ captures the scattering effects and gradient terms lead to the linear spin wave spectrum. Here $\vec{n}$ is the antiferromagnetic order parameter, $\rho_{s 0}$ is the bare spin stiffness and $c_{0}$ the bare spin wave velocity. The assumed ultraviolet cutoff is set by the lattice scale of the underlying Heisenberg model.

This model has been extensively studied in recent years using renormalization group methods [3], Monte Carlo simulations [13], and $1 / N$ expansions [5]. The results obtained using different techniques agree with each other quite well. In our calculations, we rely mostly on the $1 / N$ expansions approach. In the next Subsection, we discuss the leading order of this expansion, namely, the $N=$ $\infty$ approximation, and then proceed with the subleading $1 / N$ corrections.

\section{1. $\mathbf{N}=\infty$ Approximation}

The $N=\infty$ approximation is based on the replacement of the physical $O(3)$ quantum nonlinear $\sigma$-model, where the three-component order parameter $\vec{n}$ corresponds to three-component physical spin, by the $O(\infty)$ 
$\sigma$-model where the number of components of $\vec{n}$ is infinite. A consequence of such a replacement is the absence of damping. This makes the $N=\infty$ approximation unsuitable for such problems as the nuclear spin-lattice relaxation or the neutron scattering, where correct description of damping is essential. Nevertheless, for our particular purposes of extracting scaling parameters from the data, already the $N=\infty$ approximation is relatively accurate. It is further improved by taking into account the first subleading corrections, which are discussed in the next Subsection.

The unit length constraint can be enforced using Lagrange multiplier, $\lambda$ :

$$
\begin{gathered}
S=-\frac{\rho_{s 0}}{2} \int d^{2} \mathbf{r} \int_{0}^{1 / T} d \tau \\
\times\left[\frac{1}{c_{0}^{2}}\left(\partial_{\tau} \vec{n}\right)^{2}+(\nabla \vec{n})^{2}+i \lambda\left(\vec{n}^{2}-1\right)\right] .
\end{gathered}
$$

The action is now quadratic in $\vec{n}$ which can therefore be integrated out, leaving a functional integral for the field $\lambda$. In case of $N=\infty$, the $\lambda$ field does not fluctuate around the saddle point value $i\langle\lambda\rangle$, which in what follows is denoted as $m^{2}$ for the reason which becomes clear momentarily. By performing Fourier transform of Eq.A2, one obtains the $N=\infty$ solution for the Matsubara frequencies [5]:

$$
\chi_{s}\left(q, i \omega_{n}\right)=\frac{A}{m^{2}+c^{2} q^{2}+\omega_{n}^{2}}, \quad \xi=\frac{c}{m},
$$

where $c$ is the spin wave velocity and it is now evident that $m=c / \xi$ has the meaning of the spin wave mass, i.e. the gap in the spin wave spectrum.

The Matsubara correlator $\chi_{s}\left(q, i \omega_{n}\right)$, where $\omega_{n}=$ $2 \pi n T$, is related to the physical real frequency response function by analytical continuation: $\omega \rightarrow i \omega_{n}$. It is easy to see that Eq. (A3) corresponds to a spectrum of undamped magnons with a gap:

$$
\chi_{s}(q, \omega)=\frac{A}{m^{2}+c^{2} q^{2}-\omega^{2}}=\frac{A}{2 \epsilon_{q}}\left[\frac{1}{\epsilon_{q}-\omega}+\frac{1}{\epsilon_{q}+\omega}\right],
$$

where the spin wave dispersion is:

$$
\epsilon_{q}=\left(m^{2}+c^{2} q^{2}\right)^{1 / 2} .
$$

The imaginary part of $\chi(q, \omega)$, which describes dissipation, is:

$$
\chi_{s}^{\prime \prime}(q, \omega)=\frac{A}{2 \epsilon_{q}}\left[\delta\left(\omega-\epsilon_{q}\right)-\delta\left(\omega+\epsilon_{q}\right)\right] \quad \text { at } \quad N=\infty .
$$

Of course, for the physical $O(3)$ QNL $\sigma$ model the damping is finite and the peaks in $\chi^{\prime \prime}$ around $\omega= \pm \epsilon_{q}$ acquire finite width. This effect is captured only in the terms beyond the $N=\infty$ approximation.

Our considerations for the $\mathrm{S}=1 / 2$ and $\mathrm{S}=1$ Heisenberg models are based on the high temperature series data for the q-dependent static susceptibility of the staggered order parameter $(\vec{n})$ field , $\chi_{s}(q)$, and equal time structure factor of the same field, $S(q)$. Using Eq.(A3), one obtains:

$$
\begin{gathered}
\chi(q)=\chi_{s}\left(q, \omega_{n}=0\right)=\frac{A}{\epsilon_{q}^{2}}, \\
S(q)=T \sum_{n=-\infty}^{+\infty} \chi_{s}\left(q, i \omega_{n}\right)=\frac{A}{\epsilon_{q}} \tanh ^{-1}\left[\frac{\epsilon_{q}}{2 T}\right],
\end{gathered}
$$

where

$$
A=\chi_{s}(0) \times m^{2}, \quad \epsilon_{q}=\left(m^{2}+c^{2} q^{2}\right)^{1 / 2} .
$$

It follows directly from Eq. A7 that:

$$
\frac{\chi_{s}(0)}{\chi_{s}(q)}=1+\left(\frac{c}{m}\right)^{2} q^{2} \quad \text { for } N=\infty .
$$

Further, the ratio of Eq.A7) and Eq. A8 depends only on $\epsilon_{q} / T$ :

$$
\frac{S(q)}{T \chi_{s}(q)}=\frac{\epsilon_{q}}{2 T} \tanh ^{-1}\left[\frac{\epsilon_{q}}{2 T}\right] .
$$

We now introduce a function $\gamma[x]$ defined as:

$$
\gamma\left[\frac{x / 2}{\tanh (x / 2)}\right] \equiv x, \quad \text { for } \quad x>1,
$$

which has the following asymptotic behaviors: $\gamma[x] \simeq$ $2 \sqrt{3(x-1)}$ for $0<x-1 \ll 1$ and $\gamma[x] \simeq 2 x$ for $x \gg 1$. Then

$$
\gamma^{2}\left[\frac{S(q)}{T \chi_{s}(q)}\right]=\left(\frac{m}{T}\right)^{2}+\left(\frac{c}{T}\right)^{2} q^{2} \quad \text { for } N=\infty .
$$

\section{2. $1 / N$ Expansion}

In this Subsection, we discuss corrections to the $N=$ $\infty$ equations (A10, A13) for the physical case of $N=3$. We utilize the results of the $1 / N$ expansion calculations by Chubukov, Sachdev, and Ye [5] and find that Eqs. A13, A10 remain quite accurate even for $N=3$.

We turn first to Eq. A13). While the full q-dependence of $1 / N$ corrections have not been calculated so far, the results for small wavevectors as presented in [5] turn out to be quite sufficient for our purposes. In the asymptotic high temperature (quantum critical) regime, $m / T$ is temperature independent, while $\chi_{s}(0) / \chi_{s}(q)$ and $S(q) / T \chi_{s}(q)$ depend only on the product $q \xi \sim c q / T$. 
With straightforward application of the results of [5], we obtain for small $q$ :

$$
\begin{gathered}
\frac{\chi_{s}(0)}{\chi_{s}(q)}=1+\left(v_{1} \frac{c}{m}\right)^{2} q^{2} \\
\gamma^{2}\left[\frac{S(q)}{T \chi_{s}(q)}\right]=\left(u_{2} \frac{m}{T}\right)^{2}+\left(v_{2} \frac{c}{T}\right)^{2} q^{2},
\end{gathered}
$$

where

$$
\begin{aligned}
& v_{1}=1-0.0042 / N \rightarrow 0.9986, \\
& u_{2}=1+0.1473 / N \rightarrow 1.0491, \\
& v_{2}=1+0.0382 / N \rightarrow 1.0127
\end{aligned}
$$

\section{$(1 / N$ terms are evaluated for $N=3)$.}

The $1 / N$ corrections to the coefficients $v_{1}, v_{2}$, which enter in parenthesis in front of $q^{2}$ in Eqs. A14 A15, are essentially negligible. Higher terms in the expansion in powers of $q^{2}$ have not been calculated so far. However, once the leading terms have very small corrections, corrections to higher order terms in the expansion in $q^{2}$ should be of the same order of magnitude. The reason for this conclusion is as follows. Eqs. A14, A15) can be considered as relationshps between the position of the closest to the origin singuliarity for imaginary wavevectors, $q_{\text {sing }}=1 / \xi$, and the expansion in powers of $q^{2}$ near $q=0$. Were the higher order terms large, the position of the singuliarity expressed in terms of the coefficient in front of $q^{2}$, namely, $v_{1}$ or $v_{2}$, would change, which in its turn would lead to large corrections to $v_{1}, v_{2}$. Given the fact that such corrections are in fact small, and ignoring the possibility of accidental cancellations for both Eqs.(A14, A15), we arrive to the conclusion that higher order $q^{2 n}$ terms are small and therefore Eqs. A14, A15) are applicable for $q a \ll 1$, not only for the much narrower wavector range $q \xi \ll 1$.

It is quite remarkable that the $1 / N$ correction to the coefficient $u_{2}$ is small even when evaluated at $N=3$. Indeed, the ratio $S(\mathbf{Q}) / T \chi(\mathbf{Q})$ reflects the distribution of the spectral weight over frequencies. At the commensurate wavevector, the character of this distribution changes from a delta-function peak at finite $\omega=m$ to a smooth peak centered at $\omega=0$ as one goes from $N=\infty$ to $N=3$, which in general could have led to large $1 / N$ corrections to $u_{2}$. The fact that the rigorously calculated correction (A16) is in fact small shows that already the $N=\infty$ approximation accurately captures the relationship between the spin wave mass and the relative correlation strength at different Matsubara frequencies.

Further, since the above corrections are essentially negligible in the $\mathrm{QC}$ regime where they have been calculated, it iseems reasonable to conjecture that they may not increase drastically as $m / T$ decreases, in which case they should apply to the RC-QC crossover regime as well. The rigorous calculations to check this conjecture are prohibitively complicated and therefore have not been carried out. Instead, in the next Section we verify Eqs. A14, A15 are in agreement with the numerical data for Heisenberg models for all temperatures studied, and thereby establish that the above conjecture is indeed correct, which greatly simplifies futher considerations.

To summarize, in this Appendix we derived surprisingly simple Eqs. A14, A15, A16). Negligible (for $v_{1}, v_{2}$ ) or small $\left(\sim 5 \%\right.$ for $\left.u_{2}\right) 1 / N$ corrections to the $N=\infty$ result indicate a well behaved large $N$ expansion, and we expect the above equations to be accurate for the physical $O(3)$ model. The correspondence between the Heisenberg and sigma-models is such that $\chi_{s}(q, \omega)$ for the $\sigma$-model is $\chi(|\mathbf{q}-\mathbf{Q}|, \omega)$ for the Heisenberg model, where $\mathbf{Q}=(\pi, \pi)$. Taking this into account, we rewrite Eqs. A14, A15) in the form appropriate for the Heisenberg models, Eqs.(12, 13), and use them in Section III] for comparisons with the numerical data.

\section{APPENDIX B: CORRELATION LENGTH}

In this Appendix, we verify our resluts for $m$ and $c$ by comparing the ratio $c / m$ with the independently calculated correlation length. We calculate the correlation length, defined as the inverse rate of exponential decay of spin correlator at large distances, directly using our new "imaginary wavevector" method [23]. The idea of this calculation is based on an observation that when the pair correlation function decays exponentially up to powerlaw corrections, which is the case for two-dimensional Heisenberg models, the Fourier transform of spin correlator around the ordering wavevector, $\chi\left(\mathbf{Q}+\mathbf{n}_{q} q\right)$, where $\mathbf{n}_{q}$ is a unit vector, should have a singuliarity on the imaginary $q$ axis at $q_{\text {sing }}=i / \xi$ when analytically continued to complex $q$. The position of this singuliarity can be determined through high temperature series expansions using the methods described in [23]. In Fig.7, our results for $\xi$ for the $\mathrm{S}=1 / 2$ Heisenberg model are plotted along with the earlier quantum Monte Carlo calculations by Makivic and Ding [10]; the agreement is excellent at all temperatures.

Now we can verify the equality $\xi=c / m$, which holds by the definition of $m$. In Fig. 8, we plot our directly calculated $\xi$ by a solid line, the ratio $c / m$ determined solely from Eq.(12) as hollow circles, and the ratio of $c$ and $m$ with both determined seperately from Eq.13) as solid circles. The agreement between all three sets of data is excellent for both $S=1$ and $S=1 / 2$. This agreement not only indicates that our calculations of $m$ and $c$ are accurate, but also serves as another verification that the $\sigma$-model corectly describes wavevector and frequency dependences of the dynamical susceptibility of the Heisenberg models in the studied temperature range.

FIG. 1. $\chi(\mathbf{Q}) / \chi(\mathbf{q})$ is plotted as a function of $|\mathbf{q}-\mathbf{Q}|^{2}$ for the $\mathrm{S}=1 / 2$ (a) and $\mathrm{S}=1$ (b) Heisenberg models for selected temperatures. Linear dependences, in agreement with those derived for the $\sigma$-model Eq.(12), are observed. 
FIG. 2. $\quad \gamma^{2}[S(\mathbf{Q}) / T \chi(\mathbf{q})]$ is plotted as a function of $|\mathbf{q}-\mathbf{Q}|^{2}$ for the $\mathrm{S}=1 / 2$ (a,aa) and $\mathrm{S}=1$ (b) Heisenberg models for selected temperatures. The dependence is linear, in agreement with those derived for the $\sigma$-model Eq.(13). For the $\mathrm{S}=1 / 2$ model the intercept at $|\mathbf{q}-\mathbf{Q}|=0$ is nearly temperature independent above $T=0.6 \mathrm{~J}$, and is in agreement with the known universal QC prediction $\simeq 1.19$, shown as an arrow. For temperatures lower than $T=0.5 J$ for $\mathrm{S}=1 / 2$, and for all temperatures for $S=1$, the intercept is smaller than the $\mathrm{QC}$ prediction and decreases as the temperature decreases, indicating crossover to RC behavior.

FIG. 3. A drawing of the Brillouin zone for the square lattice; shaded area shows the wavevector range where spin correlations of the Heisenberg models are described by the $\sigma$-model.

FIG. 4. The ratio $m / T$ is plotted for (a) the $\mathrm{S}=1 / 2$, (b) $\mathrm{S}=1$ and (c) the two-plane Heisenberg models versus $T / J$ along with the known RC (Eq. (19), dot-dashed line) and QC (Eq.(21), dashed line) universal predictions. The comparisons do not contain any adjustable parameters, because the spin stiffness entering the RC expression is known from earlier studies 14, 16]. For the "more classical" $\mathrm{S}=1$ model renormalized classical behavior, and for the "critical" two plane model quantum critical behavior, are observed at all temperatures. In contrast, for the $\mathrm{S}=1 / 2$ model a crossover from $\mathrm{RC}$ to $\mathrm{QC}$ behavior occurs around $T \simeq 0.55 J$.

FIG. 5. $m / T$ is plotted versus the ratio $T /\left(c_{0} / a\right)$ for the $\mathrm{S}=1 / 2$ (circles) and $\mathrm{S}=1$ (diamonds) single-plane models, as well as the model of two $\mathrm{S}=1 / 2$ planes (triangles) coupled such that the system is at the critical point (as determined by Sandvik and Scalapino) and therefore should exhibit QC behavior at all temperatures. The difference between models in the temperature range where scaling applies is evident: the $\mathrm{S}=1$ model is always renormalized classical, the two plane model is always quantum critical, and the $\mathrm{S}=1 / 2$ model exhibits a crossover between the two around $T=0.5 \mathrm{~J}$. Moreover, for $\mathrm{S}=1 / 2$ and $\mathrm{S}=1$ models $m / T$ deviates from the respective scaling temperature dependences at roughly the same value of $T /\left(c_{0} / a\right)>0.6$ (shown as shaded area), as expected given the similar character of deviations from linearity in their spin wave spectrum (see text). For $c_{0}$, we use the spin wave theory results: $c_{0}=1.67 \mathrm{~J}$ for $\mathrm{S}=1 / 2, c_{0}=3.07 \mathrm{~J}$ for $\mathrm{S}=1$ [16], and $c_{0}=1.9 \mathrm{~J}$ for the two-plane model [17].

FIG. 6. The spin wave velocity plotted as a function of temperature for the $\mathrm{S}=1 / 2$ (a) and $\mathrm{S}=1$ (b) Heisenberg models. Dashed line shows the known $\mathrm{T}=0$ values, $c_{0}$ [18,16, and solid line is the result of $1 / S$ calculation by Kaganov and Chubukov [19], Eq.24. The temperature dependence for $T>J S$ arises from nonuniversal lattice corrections.
FIG. 7. The correlation length determined using our new method of analytical continuation to imaginary wavevectors (solid line) plotted along with earlier Monte Carlo results or Makivic and Ding 10 (circles).

FIG. 8. The correlation length for the $\mathrm{S}=1$ Heisenberg model calculated directly using the analytical continuation to imaginary wavevectors (solid line), and independently using the mapping to the quantum nonlinear $\sigma$-model, from Eq.(12) (triangles) and Eq.(13) (circles). All three sets of data agree with each other for both $S=1 / 2$ and $S=1$. 


\title{
Scaling Regimes, Crossovers, and Lattice Corrections in 2D Heisenberg Antiferromagnets
}

\author{
Norbert Elstner*, Rodney L. Glenister and Rajiv R.P. Singh \\ Department of Physics, University of California, Davis, CA 95616 \\ Alexander Sokol \\ Department of Physics and Frederick Seitz Materials Research Laboratory, \\ University of Illinois at Urbana-Champaign, Urbana, IL 61801 \\ and L.D. Landau Institute, Moscow, Russia
}

(September 8, 1994)

\begin{abstract}
We study scaling behavior in 2D, square lattice, $S=1 / 2$ and $S=1$ Heisenberg antiferromagnets using the data on full q-dependences of the equal time structure factor and the static susceptibility, calculated through high temperature series expansions. We also carry out comparisons with a model of two coupled $\mathrm{S}=1 / 2$ Heisenberg planes with the interlayer exchange coupling tuned to the $T=0$ critical point (two-plane model hereafter). For both $\mathrm{S}=1 / 2$ and $\mathrm{S}=1$ models, we separately determine the spin-wave velocity $c$ and mass $m=c / \xi$, in addition to the correlation length, $\xi$, and find that $c$ is temperature dependent; only for temperatures below $T \lesssim J S$, where $J$ is the exchange coupling, $c$ approaches its known $T=0$ value, $c_{0}$. This non-universal lattice effect is caused by the quantum nature of spin, and is therefore not captured by the quantum nonlinear $\sigma$-model. Despite this temperature dependence of the spin-wave velocity, full $q-$ and $\omega$-dependences of the dynamical susceptibility $\chi(q, \omega)$ agree with those of the universal scaling function, computable for the $\sigma$-model, for temperatures up to $T_{0} \sim 0.6 c_{0} / a$, and their further analysis leads us to the inference that below $T_{0}$ the $\mathrm{S}=1$ model is in the renormalized classical ( $\mathrm{RC}$ ) regime, the two-plane model is in the quantum critical (QC) regime in agreement with earlier work, and the $\mathrm{S}=1 / 2$ model exhibits a RC-QC crossover, centered around $\mathrm{T}=0.55 \mathrm{~J}$. In particular, for the $\mathrm{S}=1 / 2$ model above the RC-QC crossover and for the two-plane model at all temperatures where calculated, the obtained spin wave mass $m=c / \xi$ is in excellent agreement with the known universal QC prediction, $m \simeq 1.04 T$. In contrast, for the $\mathrm{S}=1 / 2$ model below the RC-QC crossover, and for the $\mathrm{S}=1$ model at all temperatures, the behavior agrees with the exact RC expression. For all three models, nonuniversal behavior occurs above $T \sim 0.6 c_{0} / a$. Our results strongly support the conjecture by Chubukov and Sachdev that the $S=1 / 2$ model is close enough to the $T=0$ critical point to exhibit QC behavior at intermediate temperatures.
\end{abstract}

*Present address: Service de Physique Théorique, CEA-Saclay, 91191 Gif-sur-Yvette Cedex, France. 
Contents

I Introduction

2

II Overview of Renormalized Classical versus Quantum Critical Scaling Behavior

III Universal Scaling Functions

IV Scaling Regimes

A Quantum Nonlinear $\sigma$-Model . . . . . . 5

B Heisenberg Models......... 6

V Conclusion

\section{APPENDIXES}

A

Quantum Nonlinear $\sigma$-Model
$\begin{array}{ll}1 & \mathrm{~N}=\infty \text { Approximation . . . . . . . . . . . } \\ 2 & 1 / N \text { Expansion . . . . . . . . . . . . . . }\end{array}$

10

B Correlation Length

12

\section{INTRODUCTION}

The problem of antiferromagnetism in two dimensions has recently attracted much attention, in part due to its relevance to high temperature superconductivity. Since the high temperature superconducting materials are derivatives, obtained by doping, of parent insulating layered $\mathrm{S}=1 / 2$ antiferromagnets, theory of the insulating phase is important for understanding the unusual magnetic and transport properties in the normal state of doped materials. Our results are directly relevant to the following two materials: $\mathrm{La}_{2} \mathrm{CuO}_{4}$, which is the insulating parent compound of the $\mathrm{La}_{2-x} \mathrm{Sr}_{\mathrm{x}} \mathrm{CuO}_{4}$ and $\mathrm{La}_{2-x} \mathrm{Ba}_{\mathrm{x}} \mathrm{CuO}_{4}$ superconductor families, and $\mathrm{Sr}_{2} \mathrm{CuO}_{2} \mathrm{Cl}_{2}$, which is unrelated to any superconducting family, but has well studied magnetic properties, similar to those of $\mathrm{La}_{2} \mathrm{CuO}_{4}$. Both materials experience antiferromagnetic transitions to the Neel state with nonzero staggered magnetization, $\mathrm{La}_{2} \mathrm{CuO}_{4}$ at $T_{N} \simeq 300 \mathrm{~K}$ and $\mathrm{Sr}_{2} \mathrm{CuO}_{2} \mathrm{Cl}_{2}$ at $T_{N} \simeq 256 \mathrm{~K}$. According to the Hohenberg-Mermin-Wagner theorem, in genuine two dimensional antiferromagnets the long range order at finite temperatures is prevented by thermal fluctuations. In real materials, small interlayer exchange interaction allows the transition to occur at a finite temperature $T_{N}$. In what follows we discuss only the properties well above $T_{N}$, where the magnetic subsystem can be considered two-dimensional.

It is well established [1] that the magnetic properties of $\mathrm{La}_{2} \mathrm{CuO}_{4}$ and $\mathrm{Sr}_{2} \mathrm{CuO}_{2} \mathrm{Cl}_{2}$ are described by the $S=1 / 2$ Heisenberg model on the two-dimensional square lattice. The Heisenberg model is defined by the following Hamiltonian:

$$
H=J \sum_{\langle i j\rangle} \mathbf{S}_{i} \mathbf{S}_{j},
$$

where $\mathbf{S}_{i}$ are local spin operators and the notation $\sum_{\langle i j\rangle}$ means that the sum is taken over all pairs of nearest neighbors. In what follows we set $k_{B}=\hbar=a=1$, where $a$ is the lattice constant, although in certain expressions we retain $a$ for clarity. The dynamical spin susceptibility $\chi(\mathbf{q}, \omega)=\chi^{\prime}+i \chi^{\prime \prime}$, is defined such that the magnetization induced by a wavevector and frequency dependent magnetic field is:

$$
\mathbf{M}(\mathbf{q}, \omega)=\chi(\mathbf{q}, \omega) \mathbf{H}_{e x t}(\mathbf{q}, \omega)
$$

We calculate high temperature series expansions in powers of $\beta=J / T$ for two quantities: the static q-dependent susceptibility, defined as a response to a static, but generally nonuniform, magnetic field,

$$
\chi(\mathbf{q}) \equiv \chi(\mathbf{q}, \omega=0)
$$

and the Fourier transform of the equal time correlation function of two spin operators,

$$
S(\mathbf{q})=\sum_{r}\left\langle\mathbf{S}_{z}(\mathbf{0}) \mathbf{S}_{z}(\mathbf{r})\right\rangle \exp (i \mathbf{q r})
$$

where angular brackets represent thermal averages. These quantities can be expressed in terms of $\chi^{\prime \prime}(\mathbf{q}, \omega)$, which plays the role of the spectral weight for spin fluctuations, according to:

$$
\begin{array}{r}
S(\mathbf{q})=T \sum_{n=-\infty}^{+\infty} \chi\left(\mathbf{q}, i \omega_{n}\right)=\frac{1}{2 \pi} \int_{-\infty}^{\infty} d \omega \frac{\chi^{\prime \prime}(\mathbf{q}, \omega)}{\operatorname{th}(\omega / 2 T)} \\
\chi(\mathbf{q})=\chi\left(\mathbf{q}, i \omega_{n}=0\right)=\frac{1}{\pi} \int_{-\infty}^{\infty} d \omega \frac{\chi^{\prime \prime}(\mathbf{q}, \omega)}{\omega}
\end{array}
$$

where $\omega_{n}=2 \pi n T$ is the imaginary bosonic Matsubara frequency.

Total spin conservation in the model leads to the relation

$$
\chi_{0}=g^{2} \mu_{B}^{2} \chi(\mathbf{q}=0)=g^{2} \mu_{B}^{2} T^{-1} S(\mathbf{q}=0),
$$

where $\chi_{0}$ is the bulk magnetic susceptibility, $g$ the electron gyromagnetic ratio, and $\mu_{B}$ the Bohr magneton.

We study both the case of $S=1 / 2$, which corresponds to $\mathrm{La}_{2} \mathrm{CuO}_{4}$ and $\mathrm{Sr}_{2} \mathrm{CuO}_{2} \mathrm{Cl}_{2}$, and the case of $S=1$. We also study a model of two $\mathrm{S}=1 / 2$ coupled Heisenberg planes at its critical point, $J_{2} / J_{1} \simeq 2.51 \pm 0.02$ [2]. For both $\chi(\mathbf{q})$ and $S(\mathbf{q})$, we are able to generate series for an arbitrary wavevector $\mathbf{q}$ complete to order $\beta^{14}$ for the $S=1 / 2$ model, and to $\beta^{10}$ for the $S=1$ model. For the two-plane model we set $J_{2} / J_{1}=2.5$, and generate series for the structure factor and the susceptibility at the antiferromagnetic ordering vector to order $\beta^{10}$. We use Pade approximation techniques for series analysis. We emphasize that the series converges extremely well in the 
temperature region presented in the paper. In fact, with the available long series, most of the Pade approximants are within a per cent, or so, from each other, down to $T \approx 0.4 J$ for $S=1 / 2$ and $T \approx 1.5 J$ for $S=1$. This allows us to put quite small errorbars on our results for all but the lowest temperatures. At the lowest temperatures, the approximants begin to significantly differ from each other and hence we do not go to still lower temperatures.

\section{OVERVIEW OF RENORMALIZED CLASSICAL VERSUS QUANTUM CRITICAL SCALING BEHAVIOR}

Scaling behavior of the 2D Heisenberg models follows from their mapping [3] to the quantum nonlinear sigma model ( $\sigma$-model hereafter). When the long range order is present at $T=0$, fluctuations at low enough temperatures are purely classical [3], in other words the characteristic frequency of spin fluctuations $\bar{\omega} \ll T$. This happens because for $T \ll \rho_{s}$ ( $\rho_{s}$ is the $T=0$ spin stiffness),

$$
\bar{\omega} \sim T^{1 / 2} \exp \left(-2 \pi \rho_{s} / T\right)
$$

decreases faster than the temperature as the temperature decreases. Accordingly, this regime is called the renormalized classical ( $\mathrm{RC}$ ) regime, where "renormalized" means that the $T=0$ parameters, such as the spin stiffness, $\rho_{s}$, and spin wave velocity, $c$, are renormalized compared to their mean-field values as a result of quantum fluctuations at shorter wavelengths.

Recently, an asymptotic expression for the correlation length has been derived for this case including the exact value of the prefactor [4]:

$$
\xi=\frac{e}{8} \frac{c}{2 \pi \rho_{s}} \exp \left(\frac{2 \pi \rho_{s}}{T}\right)\left(1-\frac{T}{4 \pi \rho_{s}}+O\left[\frac{T^{2}}{\rho_{s}^{2}}\right]\right),
$$

where $e$ is the natural logarithm base. Here $\xi$ is defined such that the dominant behavior of the pair correlation function at large distances is $\exp (-r / \xi)$ with power-law corrections.

The quantum critical ( $\mathrm{QC}$ ) regime is the high temperature scaling regime in the phase diagram of the $\sigma$-model, derived by Chakravarty, Halperin and Nelson [3], where the dominant energy scale is set by the temperature:

$$
\bar{\omega} \sim T
$$

The crossover between $\mathrm{RC}$ and $\mathrm{QC}$ regimes occurs at $T \sim$ const $\times \rho_{s}$.

As the temperature increases even further, the assumptions behind the mapping of the Heisenberg model to the $\sigma$-model eventually become invalid, which at temperatures $T \gtrsim c / a$ would destroy scaling altogether.

Chubukov and Sachdev [5] recently argued that crossover from RC to QC regime occurs around $T=0.4 \mathrm{~J}$ for the $S=1 / 2$ model. According to their paper, in the region of $T=0.4 J-0.6 J$, the system exhibits $\mathrm{QC}$ behavior. The conclusions in [5] were based on the agreement of quantum critical scaling predictions for the bulk susceptibility, $\chi_{0}$, and the NMR spin-lattice relaxation rate, $1 / T_{1}$, with the experimental measurements $[6,7]$ in $\mathrm{La}_{2} \mathrm{CuO}_{4}$ and with numerical calculations for the Heisenberg model.

However, the temperature dependence of the correlation length did not appear to support this picture of the QC behavior at intermediate temperatures [8]. $\xi(T)$ has been measured by means of inelastic neutron scattering in both $\mathrm{La}_{2} \mathrm{CuO}_{4}[9]$ and $\mathrm{Sr}_{2} \mathrm{CuO}_{2} \mathrm{Cl}_{2}$ [8], and calculated numerically for the 2D Heisenberg model using Monte-Carlo simulations [10] and series expansions approaches [11]. All experimental measurements and numerical results agree well with each other, and the $\mathrm{RC}$ expression seems to describe well the combined experimental and numerical data for the correlation length not only for $T \lesssim \rho_{s}$ [3], but even for temperatures as high as $T=J \sim 5 \rho_{s}$ [8]. At $T=J$, the second term in the expansion in powers of $T / \rho_{s}$ is only about two times smaller than the first term, therefore Eq.(8) may remain accurate to all orders only if the coefficients in front of higher terms in the series in $T / \rho_{s}$ are anomalously small. Since these coefficients are presently unknown and may indeed turn out to be small, on the basis of the correlation length data alone one would be tempted to conclude that the system remains in the $\mathrm{RC}$ regime for temperatures up to $T=J$, which would also imply an absence of a QC regime for this model [8].

The main thesis of the present paper is that such a conclusion is not justified if one examines the detailed wave-vector and temperature dependences of $S(\mathbf{q})$ and $\chi(\mathbf{q})$. It has been shown in our earlier paper [12] that the scaling functions for the static susceptibility $\chi(\mathbf{q})$, and equal time correlation function, $S(\mathbf{q})$, in the temperature range $T=0.6 J-J$ are that of the $\sigma$-model in the $\mathrm{QC}$ rather than the $\mathrm{RC}$ regime. In that temperature range, we also obtained various universal dimensionless ratios in excellent agreement with their respective $\mathrm{QC}$ predictions, with the RC predictions being far outside the errorbars.

However, the temperature dependence of the correlation length still presented a puzzle. In the quantum critical region, the leading temperature dependence of the correlation length is of the form

$$
\xi=X_{\infty}^{-1} \frac{c}{T},
$$

where $c$ is the spin-wave velocity and $X_{\infty}$ is a universal constant. With the known value of $X_{\infty}[3,5,13]$ and the known zero temperature value of the spin-wave velocity, the temperature dependence of the correlation length did not fit Eq.(10) in any extended temperature region, although the magnitude of $\xi$ around $T \sim J / 2$ is in agreement with Eq.(10). In Ref. [12], we conjectured that there are nonuniversal lattice corrections at intermediate temperatures, which affect such quantities as the correlation length, but nevertheless the scaling functions and 
the characteristic frequency of spin fluctuations are that of the QC regime.

In the present paper, we are able to show that the disagreement of the correlation length with the quantum critical prediction is indeed a result of lattice corrections, more specifically corrections to the spin wave velocity, which is temperature dependent especially at higher temperatures. We further show that when Eq.(10) is evaluated using the correct $c(T)$ rather than its $T=0$ value, the agreement with the $\mathrm{QC}$ behavior is observed for temperatures above $T \sim 0.55 J$.

\section{UNIVERSAL SCALING FUNCTIONS}

The first stage of our analysis is to establish whether or not, and in what temperature range, universal $q-$ and $\omega$-dependences of $\chi(\mathbf{q}, \omega)$, derived for the quantum nonlinear $\sigma$-model, apply to the $\mathrm{S}=1 / 2$ and $\mathrm{S}=1$ square lattice Heisenberg antiferromagnets. Here, it is convenient to introduce the spin wave mass defined as

$$
m(T)=\frac{c(T)}{\xi(T)},
$$

which, in what follows, replaces $\xi(T)$ as a scaling parameter. We emphasize that both $c(T)$ and $\xi(T)$ are measurable thermodynamic quantities. This replacement facilitates scaling comparisons: we will show that when at temperatures $T \sim J S$ the spin wave velocity becomes temperature dependent due to lattice corrections to $c$, the behavior of $m$ remains universal.

For the $\sigma$-model calculations, we employ $1 / N$ expansion approach and utilize some of the results obtained earlier by Chubukov and Sachdev [5]. We do not calculate the frequency dependence of the dynamical susceptibility explicitly. Instead, we base our comparisons on the calculated difference between the equal time correlators and static susceptibilities at all wavevectors, which is a sensitive probe of the frequency distribution of the spectral weight. Our calculations are discussed in detail in Appendix A, where we derive the following equations which relate $S(\mathbf{q})$ and $\chi(\mathbf{q})$, computable via series expansions for the Heisenberg models, to the scaling parameters $m$ and $c$ :

$$
\begin{gathered}
\frac{\chi(\mathbf{Q})}{\chi(\mathbf{q})}=1+\left(\frac{c}{m}\right)^{2}|\mathbf{q}-\mathbf{Q}|^{2}, \\
\gamma^{2}\left[\frac{S(\mathbf{q})}{T \chi(\mathbf{q})}\right]=\left(u_{2} \frac{m}{T}\right)^{2}+\left(\frac{c}{T}\right)^{2}|\mathbf{q}-\mathbf{Q}|^{2}
\end{gathered}
$$

where

$$
u_{2} \simeq 1+0.1473 / N=1.0491
$$

and the function $\gamma[x]$ is defined according to:

$$
\gamma\left[\frac{x / 2}{\tanh (x / 2)}\right] \equiv x, \quad \text { for } \quad x>1
$$

Here the expression for $u_{2}$ is evaluated at $N=3$, which corresponds to the physical three-component spin. As discussed in Appendix A, these equations are not exact. In particular, there are nonzero higher order terms in $|\mathbf{q}-\mathbf{Q}|^{2}$ and multiplicative factors in front of $c$. However, such corrections are found to be less than $1-2 \%$, which greatly simplifies further considerations.

Eqs. $(12,13)$ are in a form that greatly facilitates comparison with the numerical data: the left hand parts of the above equations are computable numerically for the Heisenberg models, and their linear dependence on $|\mathbf{q}-\mathbf{Q}|^{2}$, expected from Eqs.(12,13), can be verified by plotting on the appropriate scale. In the temperature range where the expected linear behavior is indeed observed, the scaling parameters $m$ and $c$ can be determined independently from the intercept and slope of the plotted data.

Before we proceed with the comparisons, an important remark is in order. Since at high temperatures $\chi(\mathbf{q})$ and $S(\mathbf{q})$ do not exhibit strong $q$-dependence, their small deviations from the values at $\mathbf{Q}=(\pi, \pi)$ are always linear in $|\mathbf{q}-\mathbf{Q}|^{2}$, as required by the lattice symmetry, so that at high enough temperatures $q$-dependences in Eqs. $(12,13)$ would convey no information about scaling. The high temperature crossover to non-universal weakly interacting spins is discussed more thoroughly in the next section.

We start with Eq.(12) and plot its left hand side, calculated using high temperature series expansions, versus $|\mathbf{q}-\mathbf{Q}|^{2}$ for the $\mathbf{S}=1 / 2$ and $\mathbf{S}=1$ Heisenberg models in Fig.1. Eq.(12) predicts that the plotted data should be linear. For wavevectors $|\mathbf{q}-\mathbf{Q}| \leq 0.8$, this is indeed the case for both $\mathrm{S}=1 / 2$ and $\mathrm{S}=\overline{1}$ models in a broad range of temperatures: $T=0.4 J-J$ for $\mathrm{S}=1 / 2$, and $T=1.5 J-1.9 J$ for $\mathrm{S}=1$.

We now turn to Eq.(13) and plot its left hand side versus $|\mathbf{q}-\mathbf{Q}|^{2}$ in Fig.2. Again, as expected from Eq.(13), the plotted data is linear in the same wavevector and temperature range for both $\mathrm{S}=1 / 2$ and $\mathrm{S}=1$ models.

Both Figs.1,2 are generated with $\mathbf{q}-\mathbf{Q}$ pointing along the diagonal of the Brillouin zone. The data with $\mathbf{q}-\mathbf{Q}$ pointing along other directions is virtually identical in the same wavevector and temperature range, as expected in the isotropic $\sigma$-model. Further, a straightforward estimate shows that corrections to linear spin wave spectrum due to higher gradients, not included in the $\sigma$-model, are only about $5 \%$ for the wavevector range of interest, $|\mathbf{q}-\mathbf{Q}|<0.8$. The studied temperature range is limited from below by our ability to evaluate accurately $\chi(\mathbf{q})$ and $S(\mathbf{q})$ from our high temperature series, presently known to $\beta^{14}$ for the $S=1 / 2$ model, and to $\beta^{10}$ for the $S=1$ model.

From the slope and intercept of the linear fits to the data plotted in Fig.2, we are able to determine $c$ and $m$ separately. Since this data is used extensively in what follows, we carry out comparisons of our results for $m$ 

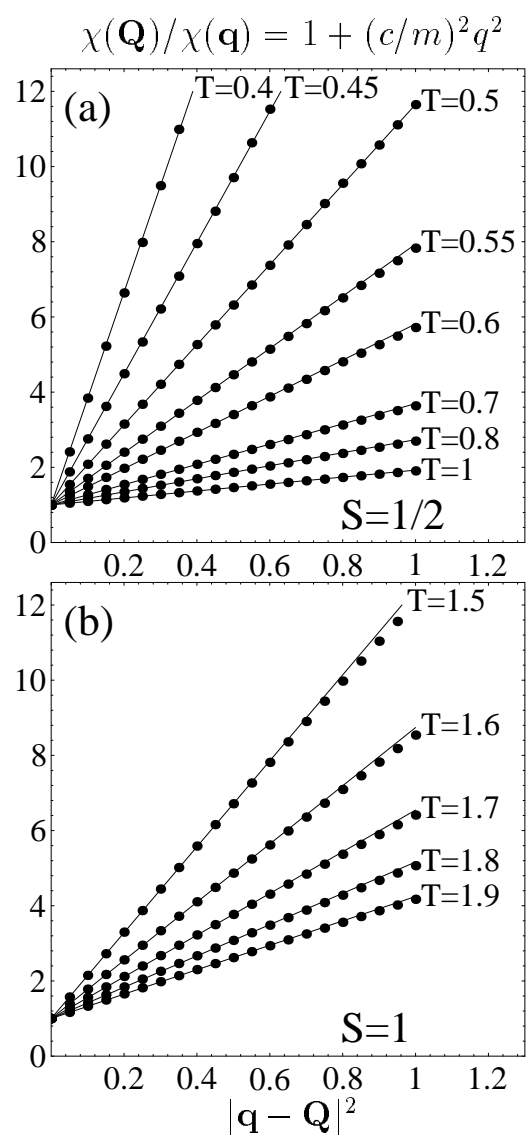

FIG. 1. $\chi(\mathbf{Q}) / \chi(\mathbf{q})$ is plotted as a function of $|\mathbf{q}-\mathbf{Q}|^{2}$ for the $S=1 / 2$ (a) and $S=1$ (b) Heisenberg models for selected temperatures. Linear dependences, in agreement with those derived for the $\sigma$-model Eq.(12), are observed.

and $c$ with direct calculations of the correlation length. The comparisons, which are described in Appendix B, confirm that our calculations are indeed accurate.

We have thereby established that Eqs.(12,13) are valid in the range $T=0.4 J-J(T=1.5 J-1.9 J)$ for $\mathrm{S}=1 / 2$ $(\mathrm{S}=1)$ for $|\mathbf{q}-\mathbf{Q}| \leq 0.8$, which means that wavevector dependences of $S(\mathbf{q})$ and $\chi(\mathbf{q})$ in the Heisenberg models are described by the $\sigma$-model in the same wavevector and temperature range, which is shaded in Fig. 3 .

\section{SCALING REGIMES}

\section{A. Quantum Nonlinear $\sigma$-Model}

We now proceed with the discussion of scaling regimes. The dynamical scaling form of the staggered correlator is $[3]$ :

$$
\chi_{s}(q, \omega)=\chi_{s}(0) \Phi^{(i)}\left(\frac{c q}{m}, \frac{\omega}{\bar{\omega}}\right)
$$
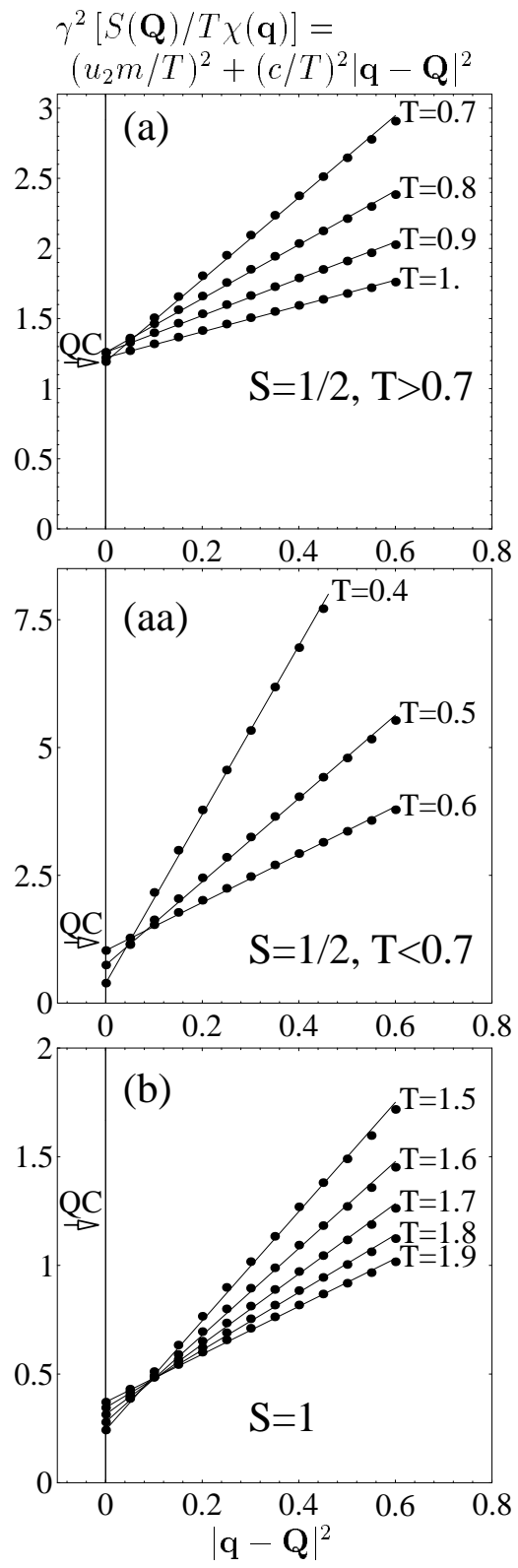

FIG. 2. $\gamma^{2}[S(\mathbf{Q}) / T \chi(\mathbf{q})]$ is plotted as a function of $|\mathbf{q}-\mathbf{Q}|^{2}$ for the $S=1 / 2$ (a,aa) and $S=1$ (b) Heisenberg models for selected temperatures. The dependence is linear, in agreement with those derived for the $\sigma$-model Eq.(13). For the $S=1 / 2$ model the intercept at $|\mathbf{q}-\mathbf{Q}|=0$ is nearly temperature independent above $T=0.6 \mathrm{~J}$, and is in agreement with the known universal QC prediction $\simeq 1.19$, shown as an arrow. For temperatures lower than $T=0.5 J$ for $\mathrm{S}=1 / 2$, and for all temperatures for $S=1$, the intercept is smaller than the QC prediction and decreases as the temperature decreases, indicating crossover to $\mathrm{RC}$ behavior.

where the scaling function for the renormalized classical regime, $\Phi^{\mathrm{RC}}$, differs from that in the quantum critical regime, $\Phi^{\mathrm{QC}}$. In general, $\bar{\omega}$ and $m$ are not equivalent: the spin wave velocity, and therefore $m$, are defined at $q \gg \xi^{-1}$, while $\bar{\omega}$ is defined at $q \sim \xi^{-1}$. In the quantum 


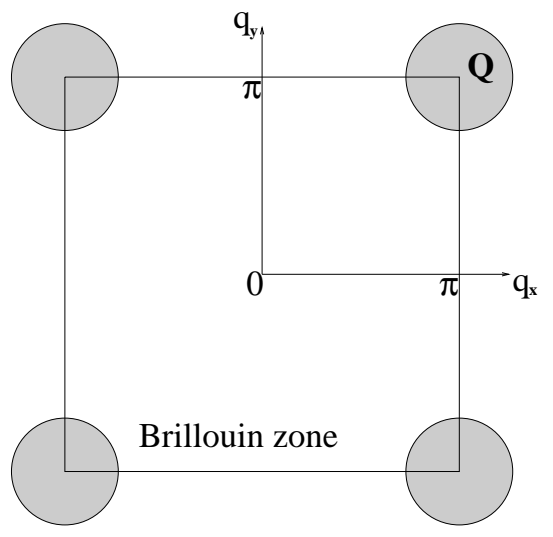

FIG. 3. A drawing of the Brillouin zone for the square lattice; shaded area shows the wavevector range where spin correlations of the Heisenberg models are described by the $\sigma$-model.

nonlinear $\sigma$-model, $c$ is expected to be temperature dependent only when the temperature is comparable to the ultraviolet cutoff $\Lambda$ (except in case of relativistic invariance of the cutoff procedure, when $c$ is always temperature independent and coincides with its bare value).

In the $\mathrm{RC}$ regime temperature dependences of the spin wave mass for $N=\infty$ and $N=3$ are different: $[3,5]$ :

$$
m \sim \exp \left(-\frac{2 \pi \rho_{s}}{T}\right) \times\left\{\begin{array}{ll}
T & \text { for } N=\infty \\
\rho_{s} & \text { for } N=3
\end{array} .\right.
$$

Furthermore, in this regime $\bar{\omega}$ is not equivalent to $m$, except for $N=\infty$ :

$$
\frac{\bar{\omega}}{m} \sim\left\{\begin{array}{ll}
1 & \text { for } N=\infty \\
\left(T / \rho_{s}\right)^{1 / 2} & \text { for } N=3
\end{array} .\right.
$$

The exact asymptotic expression for $m$ follows immediately from Eq.(8):

$$
m=\frac{16 \pi \rho_{s}}{e} \exp \left(\frac{-2 \pi \rho_{s}}{T}\right)\left(1-\frac{T}{4 \pi \rho_{s}}+O\left[\frac{T^{2}}{\rho_{s}^{2}}\right]\right)^{-1} .
$$

The value of $\rho_{s}$, which is the only dimensional parameter in Eq.(19), is known for the Heisenberg models from zero temperature series expansions [14], quantum Monte Carlo simulations [15], and 1/S calculations [16].

In the QC regime, temperature is the only energy scale in the problem, so that:

$$
\bar{\omega} \sim m \sim T,
$$

which is valid for both $N=3$ and $N=\infty$ models. Furthermore, the ratio $m / T$ is universal in the $\sigma$-model, and has been calculated via the $1 / N$ approach [5]:

$$
\frac{m}{T}=\Theta\left(1+\frac{0.2373}{N}\right) \sim 0.9624+\frac{0.2284}{N} \approx 1.04,
$$

where $\Theta=2 \log [(1+\sqrt{5}) / 2]$. Given that already the first subleading $1 / N$ correction is small $(\sim 8 \%)$ for $N=3$, Eq.(21) is likely to be close to the true value for the $O(3) \sigma$-model, and is hereafter adopted as the QC prediction. It can be compared with calculations based on other techniques:

$$
\frac{m}{T}= \begin{cases}0.9, & 2+\epsilon \text { expansions }[3] \\ 1.25 \pm .25, & \text { Monte Carlo [13] }\end{cases}
$$

The results are generally consistent with each other. Further support for the numerical value obtained in the $1 / N$ calculation comes from the study of the two-plane model at its $T=0$ critical coupling by Sandvik and Scalapino [2], where temperature dependence of the correlation length was found to be in agreement with that derived from Eq.(21).

Thus, both RC (19) and QC (21) predictions for $m(T)$ are available to carry out comparisons with Heisenberg models without any adjustable parameters.

\section{B. Heisenberg Models}

We start by comparing behavior of the spin wave mass for $\mathrm{S}=1 / 2, \mathrm{~S}=1$, and the two plane models. The $\mathrm{S}=1$ model, due to its larger spin, is "more classical" than the $\mathrm{S}=1 / 2$ model, hence it is less likely to have a range of quantum critical behavior. On the other hand, for the two plane model at its critical point, temperature dependences of $\chi_{0}$ and $\xi$ consistent with the QC regime had been reported earlier by Sandvik and Scalapino [2]. The comparison of different models proves quite informative.

We now plot $m(T)$ for these three models along with the universal RC (19) and QC (21) scaling predictions in Fig.4. It is evident from Fig.4 that $m(T)$ for the $\mathrm{S}=1$ model shows RC behavior at all temperatures, for the two plane model QC behavior at all temperatures, and for the $\mathrm{S}=1 / 2$ single plane model a crossover between RC behavior below $T \sim 0.45 J$ and $\mathrm{QC}$ behavior above $T=0.65 J$.

We now discuss the onset of nonuniversal behavior at high temperatures. The temperature scale at which nonuniversal behavior arises is set roughly by $c / a$. The spin wave spectrum becomes nonlinear at $q \sim a^{-1}$, and the excitations in this wavevector range are suppressed only when $T \ll c / a$. The spin stiffness, on the other hand, is less relevant to this crossover to nonuniversal behavior at high temperatures. To a crude approximation we expect scaling to fail for $\mathrm{S}=1 / 2$ and $\mathrm{S}=1$ models at $T /(c / a)$ of the same order, given their similar spin wave spectrum. In order to study failure of scaling, we therefore plot the ratio $m / T$ versus $T /\left(c_{0} / a\right)$ (Fig. 5 ). The use of $c_{0}$ instead of the temperature dependent $c(T)$ is to avoid a nonlinear temperature scale. In this particular case, the difference between $c$ and $c_{0}$ is less important given the ambiguous character of the upper boundary of scaling behavior. 

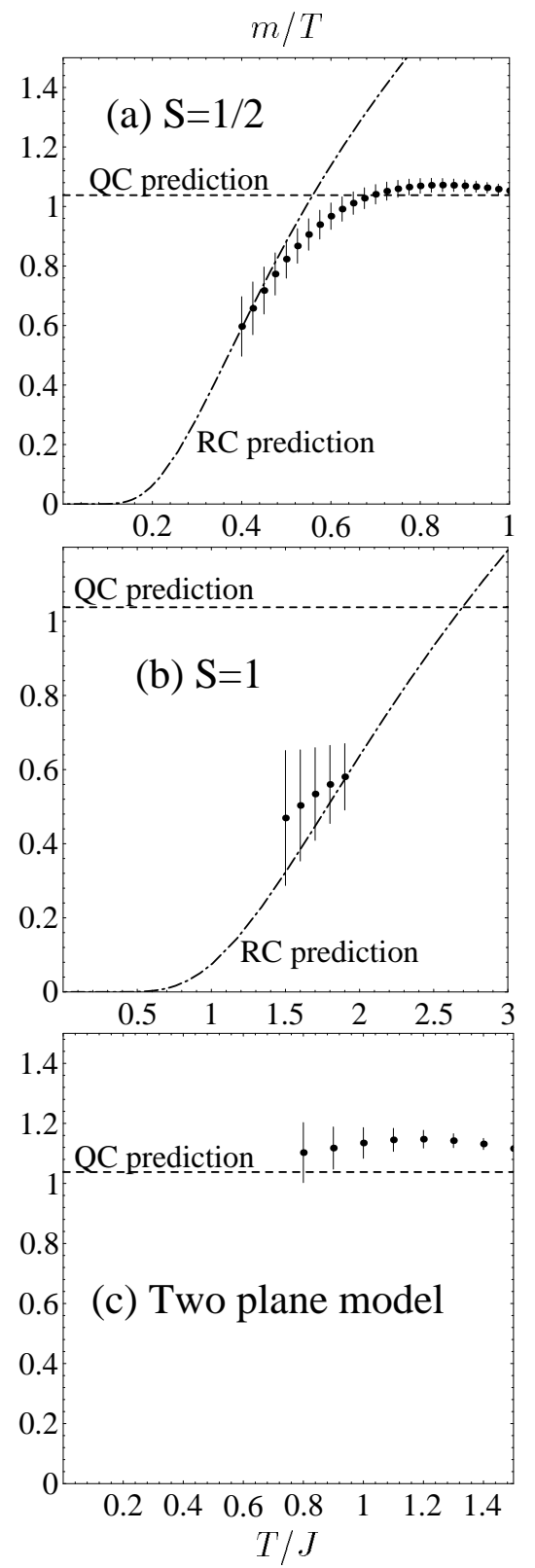

FIG. 4. The ratio $m / T$ is plotted for (a) the $\mathrm{S}=1 / 2$, (b) $\mathrm{S}=1$ and (c) the two-plane Heisenberg models versus $T / J$ along with the known RC (Eq.(19), dot-dashed line) and QC (Eq.(21), dashed line) universal predictions. The comparisons do not contain any adjustable parameters, because the spin stiffness entering the RC expression is known from earlier studies $[14,16]$. For the "more classical" $\mathrm{S}=1$ model renormalized classical behavior, and for the "critical" two plane model quantum critical behavior, are observed at all temperatures. In contrast, for the $S=1 / 2$ model a crossover from $\mathrm{RC}$ to $\mathrm{QC}$ behavior occurs around $T \simeq 0.55 J$.

One can see from Fig. 5 that deviations from the universal behavior of $m$ occurs at similar values of the ratio $T /\left(c_{0} / a\right) \sim 0.6$ for both $\mathrm{S}=1 / 2$ and $\mathrm{S}=1$ models, above which a crossover to the $T \gg J$ asymptotics $m \sim 1 / T$ occurs. The region where $m$ becomes nonuniversal and no longer represents the spin wave mass is shaded

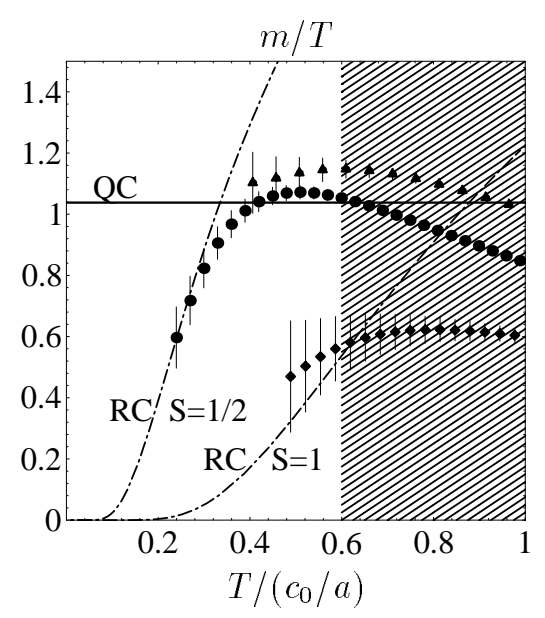

FIG. 5. $m / T$ is plotted versus the ratio $T /\left(c_{0} / a\right)$ for the $\mathrm{S}=1 / 2$ (circles) and $\mathrm{S}=1$ (diamonds) single-plane models, as well as the model of two $\mathrm{S}=1 / 2$ planes (triangles) coupled such that the system is at the critical point (as determined by Sandvik and Scalapino) and therefore should exhibit QC behavior at all temperatures. The difference between models in the temperature range where scaling applies is evident: the $\mathrm{S}=1$ model is always renormalized classical, the two plane model is always quantum critical, and the $S=1 / 2$ model exhibits a crossover between the two around $T=0.5 \mathrm{~J}$. Moreover, for $\mathrm{S}=1 / 2$ and $\mathrm{S}=1$ models $m / T$ deviates from the respective scaling temperature dependences at roughly the same value of $T /\left(c_{0} / a\right)>0.6$ (shown as shaded area), as expected given the similar character of deviations from linearity in their spin wave spectrum (see text). For $c_{0}$, we use the spin wave theory results: $c_{0}=1.67 J$ for $\mathrm{S}=1 / 2, c_{0}=3.07 \mathrm{~J}$ for $\mathrm{S}=1$ [16], and $c_{0}=1.9 \mathrm{~J}$ for the two-plane model [17].

in Fig.5. Expressed in terms of $J$, the range where universal behavior of $m(T)$ holds is below $T \sim J$ for the $\mathrm{S}=1 / 2$ model and below $T \sim 1.9 J$ for the $\mathrm{S}=1$ model.

Now, we turn to the analysis of the spin wave velocity calculated in Sect.III, and plot $c$ determined from the data at different temperatures using Eq.13 in Fig.6.

Dashed lines show the known $T=0$ value of the spin wave velocity calculated using $1 / S$ expansions [16],

$$
\begin{array}{rl}
c=2 \sqrt{2} J & S\left(1+0.1580 / 2 S+0.0216 /(2 S)^{2}\right) \\
& \simeq \begin{cases}1.67 J & \text { for } \mathrm{S}=1 / 2 \\
3.07 J & \text { for } \mathrm{S}=1\end{cases}
\end{array}
$$

which for $\mathrm{S}=1 / 2$ compares favorably with the $\mathrm{T}=0$ series expansion calculations [14].

For all temperatures studied the spin wave velocity turns out to be temperature dependent for both $\mathrm{S}=1 / 2$ and $\mathrm{S}=1$ models. Towards the lowest temperatures in the numerically accessible range, $c$ approaches the expected $\mathrm{T}=0$ values given by Eq.(23). Since in the $\sigma$ model the spin wave velocity is temperature independent except near the ultraviolet cutoff, which in our case is set by the scale of order $c / a \sim 2 \sqrt{2} J S$, the observed 

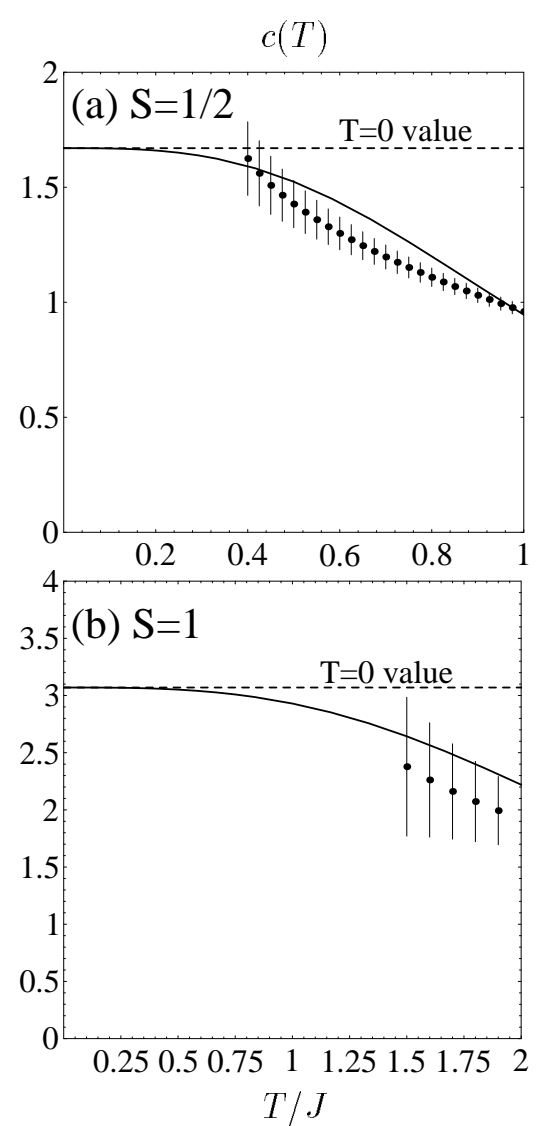

FIG. 6. The spin wave velocity plotted as a function of temperature for the $S=1 / 2$ (a) and $S=1$ (b) Heisenberg models. Dashed line shows the known $\mathrm{T}=0$ values, $c_{0}[18,16]$, and solid line is the result of $1 / S$ calculation by Kaganov and Chubukov [19], Eq.24. The temperature dependence for $T>J S$ arises from nonuniversal lattice corrections.

temperature dependent spin wave velocity clearly results from the quantum nature of spin, when the temperature becomes comparable to $J S$.

The quantum corrections to the spin wave velocity originating from higher order $1 / S$ terms in the spin wave theory, were calculated by Kaganov and Chubukov [19]:

$$
c(T)=c_{0}\left[1+\frac{\zeta(3)}{8 \pi S}\left(\frac{T}{S J}\right)^{3}\right]^{-1},
$$

where $\zeta(x)$ is the Riemann zeta function. The predictions of Eq.(24), evaluated for $S=1 / 2$ and $S=1$, are shown as solid lines in Fig.6a,b, respectively. The agreement with numerical calculations is quite good. Despite higher order terms in the expansion in powers of $T / J S$, it appears that Eq.(24) describes the observed temperature dependence of $c$ fairly well for both $S=1 / 2$ and $S=1$.

At this stage an important question is, does the temperature dependence of $c$ destroy scaling altogether? One might expect that once lattice corrections become important for quantities like the spin-wave velocity, they might also lead to a breakdown of scaling altogether by introducing deviations from linearity in the spin wave spectrum and appearance of spectral weight in modes not contained in the quantum nonlinear $\sigma$-model. We argue that this is not the case here. Indeed, from the fact that Eqs. $(12,13)$ remain valid when $c(T)$ is already temperature dependent, we conclude that the spin wave spectrum still remains linear even though $c(T)$ deviates from its $\mathrm{T}=0$ value. By the same token, we conclude that there are no modes with significant spectral weight other than those described by the $\sigma$-model.

Another universal quantity which has not been discussed in our paper so far is the overall prefactor, $\chi_{Q}$, in front of the scaling expression for $\chi(\mathbf{q}, \omega)$ in Eq.(16). This prefactor, which is renormalized by short wavelength quantum fluctuations, can be expressed in terms of $N_{0}$ and $\rho_{s}$ for temperatures where corrections of order $T / J S$ can be ignored. Where $c$ is $T$-dependent, this quantity becomes $T$-dependent as well. However, quantum corrections to the prefactor due to $1 / S$ terms have not yet been calculated analytically.

Now the reason why the quantum critical scaling fails to describe the temperature dependence of the correlation length is clear. The correlation length derived from Eq.(A3) is $\xi=\mathrm{c} / \mathrm{m}$. If one assumes that the spin wave velocity is temperature independent above $T=0.4 J$, the quantum critical expression does not agree with the numerical data. Given the discussions above, we argue that one should consider not the temperature dependence of $\xi$, but that of $m=c / \xi$, because $m$ is unaffected by the lattice corrections to $c$. Then, the crossover from $\mathrm{RC}$ behavior to QC behavior at temperatures around $J / 2$ becomes evident.

\section{CONCLUSION}

Magnetic properties of the doped superconducting cuprates may be closely related to the undoped parent compounds, such as $\mathrm{La}_{2} \mathrm{CuO}_{4}$ and $\mathrm{Sr}_{2} \mathrm{CuO}_{2} \mathrm{Cl}_{2}$, which are well described by the square lattice Heisenberg model. Motivated by this relationship, we have studied scaling and the role of lattice corrections in two dimensional square lattice Heisenberg antiferromagnets.

In [3], Chakravarty, Halperin, and Nelson mapped 2D collinear quantum antiferromagnets onto the quantum nonlinear $\sigma$-model, and derived the corresponding phase diagram. When the $\mathrm{T}=0$ ground state of the corresponding $\sigma$-model has Neel order, as is the case for the square lattice Heisenberg models, the low temperature scaling regime is classical (often called renormalized classical, or RC), where the characteristic energy scale $\bar{\omega} \ll T$, while at higher temperatures a crossover to the quantum critical (QC) regime may be observed, where $\bar{\omega} \sim T$. The $\mathrm{S}=1 / 2$ square lattice Heisenberg model, which describes $\mathrm{La}_{2} \mathrm{CuO}_{4}$, was shown to exhibit $\mathrm{RC}$ behavior at low temperatures [3]. As it has a substantial ordered moment, 
it was earlier believed not to have a region of quantumcritical behavior at intermediate temperatures.

More recently, Chubukov and Sachdev [5] argued that the crossover from the RC to nonuniversal microscopic behavior occurs through the intermediate $\mathrm{QC}$ regime at $0.4 J<T<0.6 J$, with universal behavior extending up to $T=0.6 J$ and nonuniversal behavior above that temperature. The importance of this at first sight minor difference concerning a fairly narrow temperature range, arises from the fact that upon doping the QC region was argued to expand rather rapidly, covering a much broader, and well accessible temperature range [5]. This scenario has important implications for our views of magnetism in the superconducting cuprate materials [5,20].

In our earlier paper [12], we showed that the scaling functions for the equal time spin correlator, $S(\mathbf{q})$, and the static susceptibility, $\chi(\mathbf{q})$, in the $\mathrm{S}=1 / 2$ Heisenberg model in the temperature range $T=0.6 J-J$ agree with those calculated for the $\sigma$-model in the $\mathrm{QC}$ regime. The same turned out to be true for the ratio $S(\mathbf{q}) / T \chi(\mathbf{q})$. Our comparisons did not involve any adjustable parameters.

In the present paper, we undertook a detailed study of these issues using the high temperature series expansions approach. The simultaneous study of $\mathrm{S}=1 / 2$ and $\mathrm{S}=1$ models allows us to contrast the two cases and highlight the parameter-free agreement of the intermediate temperature behavior for the $S=1 / 2$ case with the universal quantum critical predictions of the non-linear $\sigma$-model. For both $S=1 / 2$ and $S=1$ models, we find that the q-dependences of $S(\mathbf{q})$ and $\chi(\mathbf{q})$ separately, as well as of their ratio, agree very well up to $T \sim 0.6 c_{0} / a$ with the $\sigma$-model predictions calculated separately using $1 / N$ expansions (for $1 / N$ calculations, we utilized many of the results obtained in [5]). This allows us to calculate the temperature dependence of the spin wave mass, defined as $m=c / \xi$, for which both $\mathrm{RC}$ and $\mathrm{QC}$ predictions are known without adjustable parameters.

For $\mathrm{S}=1 / 2$, we find that $m(T)$ is in good agreement with the RC prediction for $T<0.45 J$, while for $0.65 J<$ $T<J$ the results are very close to the QC prediction and far from the RC prediction, exhibiting a crossover between these two regimes for $0.45 J<T<0.65 J$. Furthermore, we find that for $T>0.5 J$, the lattice corrections cause a temperature dependence of the spin-wave velocity $c$ and affect the overall prefactor in front of the scaling expression for $\chi(\mathbf{q}, \omega)$. The new results strongly support our earlier conjecture [12] that lattice corrections are such that full $\mathrm{q}^{-}$and $\omega$-dependences remain universal in the QC regime for temperatures up to $T \simeq 0.6 c_{0} / a \sim J$ :

$$
\chi(\mathbf{q}, \omega)=\chi_{Q} \Phi^{\mathrm{QC}}\left(\frac{c|\mathbf{q}-\mathbf{Q}|}{T}, \frac{\omega}{T}\right),
$$

where $\Phi^{\mathrm{QC}}(x, y)$ is the scaling function of the $\mathrm{QC}$ regime, computable using, for instance, the approach of [5]. For the $\mathrm{S}=1$ model, where quantum fluctuations are substantially less, we find that RC behavior directly crosses over to the high temperature interacting local-spin behavior without an intervening QC regime. Finally, the observed lattice corrections to $c$ for both $\mathrm{S}=1 / 2$ and $\mathrm{S}=1$ roughly agree in magnitude and temperature dependence with earlier calculations of quantum $T / J S$ effects by Kaganov and Chubukov [19].

In their recent publication, Greven and coworkers [8] pointed out the agreement of the measured and numerically calculated correlation length $\xi(T)$ with RC prediction for all temperatures below $T=J$. In our work, we present an alternative theory which is not only consistent with the same data for $\xi(T)$, but explains the $q-$ and $\omega$-dependences of spin correlators as well. We show that detailed examination of the wavevector and temperature dependence of $S(q)$ and $\chi(q)$ supports the picture of renormalized classical behavior for temperatures up to $T=0.45 J$, and a crossover to a quantum critical behavior around $T=J / 2$.

While the QC behavior for the spin wave mass occurs in our calculations in the temperature range $T>0.6 \mathrm{~J}$ currently inaccessible to experiments in $\mathrm{La}_{2} \mathrm{CuO}_{4}$ and $\mathrm{Sr}_{2} \mathrm{CuO}_{2} \mathrm{Cl}_{2}$, many signatures of the $\mathrm{QC}$ behavior have been experimentally observed at lower temperatures. For instance, the nuclear relaxation rate, $1 / T_{1}$, in $\mathrm{La}_{2} \mathrm{CuO}_{4}$ saturates above $T=600 \mathrm{~K} \sim 0.4 J[7]$ at a value close in magnitude to the universal QC prediction [5]. This is consistent with the present study, as it has been shown [5,21] that for those quantities which in the $\mathrm{RC}$ regime depend on $\xi$ through its logarithm only, such as the ratio $T_{1} T / T_{2 \mathrm{G}}$ and the bulk susceptibility, $\chi_{0}$, the $\mathrm{RC}-\mathrm{QC}$ crossover should be shifted substantially towards lower temperatures, in agreement with the measurements of $\chi_{0}[6]$ and $T_{1} T / T_{2 g}[7]$ in $\mathrm{La}_{2} \mathrm{CuO}_{4}$.

It has been proposed earlier that the temperature range of quantum critical behavior rapidly expands with doping, and that the scaling behavior described by the quantum nonlinear $\sigma$-model may be observed in doped antiferromagnets, and specifically in the high temperature superconductors $[5,20,12]$. We hope that the study of the renormalized classical to quantum critical scaling crossover and of the origin of nonuniversal corrections to scaling in the insulator presented here, will be helpful in understanding magnetic behavior of the doped systems as well.

\section{ACKNOWLEDGMENTS}

We are grateful to R.J. Birgeneau, S. Chakravarty, A.V. Chubukov, M. Greven, A.J. Millis, D. Pines, S. Sachdev, A.W. Sandvik, and D.J. Scalapino for many useful discussions. N.E. was supported by the Deutsche Forschungsgemeinschaft. R.L.G. and R.R.P.S. were supported by the NSF Grant DMR93-18537. A.S. was supported by the NSF Grant DMR89-20538 through the Frederick Seitz Materials Research Laboratory. The computations have been performed using the IBM RISC/6000 workstation received through the Shared Uni- 
versity Research Grant from the IBM Corporation. N.E. would like to acknowledge the kind hospitality of A.P. Young and Physics Department of the University of California at Santa Cruz where part of this work was done. A.S. is grateful to the Aspen Center for Physics for hospitality during intial stages of this work.

[1] For comprehensive reviews, see S. Chakravarty, in Proceedings of High Temperature Superconductivity, edited by K.S. Bedell et al. (Addison-Wesley, CA, 1990), and E. Manousakis, Rev. Mod. Phys. 63, 1 (1991).

[2] A.W. Sandvik and D.J. Scalapino, Phys. Rev. Lett., 72, 277 (1994), and unpublished. The two plane model was discussed earlier by K. Hida, J. Phys. Soc. Japan, 61, 1013 (1992); A.J. Millis and H. Monien, Phys. Rev. Lett., 70, 2810 (1993).

[3] S. Chakravarty, B.I. Halperin, and D.R. Nelson, Phys. Rev. B 39, 2344 (1989); see also S. Chakravarty and R. Orbach, Phys. Rev. Lett. 64, 224 (1990).

[4] P. Hasenfratz and F. Niedermayer, Phys. Lett. B 268, 231 (1991); Z. Phys. B 92, 91 (1993).

[5] A.V. Chubukov and S. Sachdev, Phys. Rev. Lett. 71, 169 (1993); A.V. Chubukov, S. Sachdev, and J. Ye, Phys. Rev. B49, 11919 (1994).

[6] D.C. Johnston, Phys. Rev. Lett. 62, 957 (1989); D.C. Johnston, S.K. Sinha, A.J. Jacobson and J.M. Newsam, Physica (Amsterdam), 153-155 C, 572 (1988)

[7] T. Imai, C.P. Slichter, K. Yoshimura and K. Kosuge, Phys. Rev. Lett. 70, 1002 (1993); T. Imai, C.P. Slichter, K. Yoshimura, M.Katoh and K. Kosuge, Phys. Rev. Lett. 71, 1254 (1993).

[8] M. Greven et al., Phys. Rev. Lett. 72, 1096 (1994).

[9] B. Keimer et al., Phys. Rev. B 46, 14034 (1992).

[10] H.-Q. Ding and M.S. Makivic, Phys. Rev. Lett, 64, 1449 (1990); M.S. Makivic and H.-Q. Ding, Phys. Rev. B 43 , 3662 (1990).

[11] R.R.P. Singh and R.L. Glenister, Phys. Rev. B 46, 11871 (1992).

[12] A. Sokol, R.L. Glenister and R.R.P. Singh, Phys. Rev. Lett., 72, 1549 (1994).

[13] E. Manousakis and R. Salvador, Phys. Rev. Lett., 62, 1310 (1989); Phys. Rev. B 40, 2205 (1989). Remark: in our opinion, the data for the slab thickness $N_{\tau}=2$ may not be appropriate for extracting properties of the thermodynamic limit $\left(N_{\tau}=\infty\right)$, where $m / T$ is defined. We therefore repeated the analysis of Manousakis and Salvador omitting the data for $N_{\tau}=2$, arriving to the errorbars for $m / T$, shown in Eq.(22), which are larger than the errorbars quoted in the original paper. With this larger erorbars, the result agrees with calculations in the framework of other approaches.

[14] R.R.P. Singh, Phys. Rev. B 39, 9760 (1989); R.R.P. Singh and D. Huse, Phys. Rev. B 40, 7247 (1989).

[15] U.J. Wiese and H.P. Ying, Z. Phys. B93, 147 (1994).

[16] T. Oguchi, Phys. Rev, 117, 117 (1960); S. Chakravarty and C. Castilia, Phys. Rev. B 43, 13687 (1991); J. Igarashi, Phys. Rev. B 46, 10763 (1992).

[17] A.V. Chubukov and A.W. Sandvik, unpublished.

[18] R.R.P. Singh, Phys. Rev. B47, 12337 (1993).

[19] M.I. Kaganov and A.V. Chubukov, in "Spin waves and Magnetic Excitations", part I, Eds. A.S. BorovikRomanov and S.K. Sinha, North-Holland, Amsterdam (1988).

[20] A. Sokol and D. Pines, Phys. Rev. Lett. 71, 2813 (1993).

[21] A.V. Chubukov, S. Sachdev, and A. Sokol, Phys. Rev. B 49, 9052 (1994).

[22] E.H. Fradkin, Field Theories of Condensed Matter Systems, Addison-Wesley, Redwood City, CA (1991).

[23] R.L. Glenister, N. Elstner, R.R.P. Singh, and A. Sokol, to be published.

\section{APPENDIX A: QUANTUM NONLINEAR $\sigma$-MODEL}

The mapping [3] of the low energy spectrum of the Heisenberg model to the quantum nonlinear sigma model has been extensively discussed in the literature and textbooks (e.g., see [22]). Here we briefly discuss some aspects of this mapping, and of the $1 / N$ expansion in this model, as necessary for our purposes. We refer the reader to Refs. $[3,5]$ for a far more detailed treatment.

The action of the quantum nonlinear $\sigma$-model can be written as:

$$
\begin{array}{r}
S=-\frac{\rho_{s 0}}{2} \int d^{2} \mathbf{r} \int_{0}^{1 / T} d \tau\left[\frac{1}{c_{0}^{2}}\left(\partial_{\tau} \vec{n}\right)^{2}+(\nabla \vec{n})^{2}\right] \\
\vec{n}^{2}=1
\end{array}
$$

where the constraint $\vec{n}^{2}=1$ captures the scattering effects and gradient terms lead to the linear spin wave spectrum. Here $\vec{n}$ is the antiferromagnetic order parameter, $\rho_{s 0}$ is the bare spin stiffness and $c_{0}$ the bare spin wave velocity. The assumed ultraviolet cutoff is set by the lattice scale of the underlying Heisenberg model.

This model has been extensively studied in recent years using renormalization group methods [3], Monte Carlo simulations [13], and $1 / N$ expansions [5]. The results obtained using different techniques agree with each other quite well. In our calculations, we rely mostly on the $1 / N$ expansions approach. In the next Subsection, we discuss the leading order of this expansion, namely, the $N=$ $\infty$ approximation, and then proceed with the subleading $1 / N$ corrections.

\section{1. $\mathrm{N}=\infty$ Approximation}

The $N=\infty$ approximation is based on the replacement of the physical $O(3)$ quantum nonlinear $\sigma$-model, where the three-component order parameter $\vec{n}$ corresponds to three-component physical spin, by the $O(\infty)$ 
$\sigma$-model where the number of components of $\vec{n}$ is infinite. A consequence of such a replacement is the absence of damping. This makes the $N=\infty$ approximation unsuitable for such problems as the nuclear spin-lattice relaxation or the neutron scattering, where correct description of damping is essential. Nevertheless, for our particular purposes of extracting scaling parameters from the data, already the $N=\infty$ approximation is relatively accurate. It is further improved by taking into account the first subleading corrections, which are discussed in the next Subsection.

The unit length constraint can be enforced using Lagrange multiplier, $\lambda$ :

$$
\begin{gathered}
S=-\frac{\rho_{s 0}}{2} \int d^{2} \mathbf{r} \int_{0}^{1 / T} d \tau \\
\times\left[\frac{1}{c_{0}^{2}}\left(\partial_{\tau} \vec{n}\right)^{2}+(\nabla \vec{n})^{2}+i \lambda\left(\vec{n}^{2}-1\right)\right] .
\end{gathered}
$$

The action is now quadratic in $\vec{n}$ which can therefore be integrated out, leaving a functional integral for the field $\lambda$. In case of $N=\infty$, the $\lambda$ field does not fluctuate around the saddle point value $i\langle\lambda\rangle$, which in what follows is denoted as $m^{2}$ for the reason which becomes clear momentarily. By performing Fourier transform of Eq.(A2), one obtains the $N=\infty$ solution for the Matsubara frequencies [5]:

$$
\chi_{s}\left(q, i \omega_{n}\right)=\frac{A}{m^{2}+c^{2} q^{2}+\omega_{n}^{2}}, \quad \xi=\frac{c}{m}
$$

where $c$ is the spin wave velocity and it is now evident that $m=c / \xi$ has the meaning of the spin wave mass, i.e. the gap in the spin wave spectrum.

The Matsubara correlator $\chi_{s}\left(q, i \omega_{n}\right)$, where $\omega_{n}=$ $2 \pi n T$, is related to the physical real frequency response function by analytical continuation: $\omega \rightarrow i \omega_{n}$. It is easy to see that Eq.(A3) corresponds to a spectrum of undamped magnons with a gap:

$$
\chi_{s}(q, \omega)=\frac{A}{m^{2}+c^{2} q^{2}-\omega^{2}}=\frac{A}{2 \epsilon_{q}}\left[\frac{1}{\epsilon_{q}-\omega}+\frac{1}{\epsilon_{q}+\omega}\right],
$$

where the spin wave dispersion is:

$$
\epsilon_{q}=\left(m^{2}+c^{2} q^{2}\right)^{1 / 2}
$$

The imaginary part of $\chi(q, \omega)$, which describes dissipation, is:

$$
\chi_{s}^{\prime \prime}(q, \omega)=\frac{A}{2 \epsilon_{q}}\left[\delta\left(\omega-\epsilon_{q}\right)-\delta\left(\omega+\epsilon_{q}\right)\right] \text { at } \quad N=\infty .
$$

Of course, for the physical $O(3)$ QNL $\sigma$ model the damping is finite and the peaks in $\chi^{\prime \prime}$ around $\omega= \pm \epsilon_{q}$ acquire finite width. This effect is captured only in the terms beyond the $N=\infty$ approximation.

Our considerations for the $\mathrm{S}=1 / 2$ and $\mathrm{S}=1$ Heisenberg models are based on the high temperature series data for the q-dependent static susceptibility of the staggered order parameter $(\vec{n})$ field, $\chi_{s}(q)$, and equal time structure factor of the same field, $S(q)$. Using Eq.(A3), one obtains:

$$
\begin{gathered}
\chi(q)=\chi_{s}\left(q, \omega_{n}=0\right)=\frac{A}{\epsilon_{q}^{2}}, \\
S(q)=T \sum_{n=-\infty}^{+\infty} \chi_{s}\left(q, i \omega_{n}\right)=\frac{A}{\epsilon_{q}} \tanh ^{-1}\left[\frac{\epsilon_{q}}{2 T}\right],
\end{gathered}
$$

where

$$
A=\chi_{s}(0) \times m^{2}, \quad \epsilon_{q}=\left(m^{2}+c^{2} q^{2}\right)^{1 / 2} .
$$

It follows directly from Eq.(A7) that:

$$
\frac{\chi_{s}(0)}{\chi_{s}(q)}=1+\left(\frac{c}{m}\right)^{2} q^{2} \text { for } N=\infty \text {. }
$$

Further, the ratio of Eq.(A7) and Eq.(A8) depends only on $\epsilon_{q} / T$ :

$$
\frac{S(q)}{T \chi_{s}(q)}=\frac{\epsilon_{q}}{2 T} \tanh ^{-1}\left[\frac{\epsilon_{q}}{2 T}\right]
$$

We now introduce a function $\gamma[x]$ defined as:

$$
\gamma\left[\frac{x / 2}{\tanh (x / 2)}\right] \equiv x, \quad \text { for } \quad x>1
$$

which has the following asymptotic behaviors: $\gamma[x] \simeq$ $2 \sqrt{3(x-1)}$ for $0<x-1 \ll 1$ and $\gamma[x] \simeq 2 x$ for $x \gg 1$. Then

$$
\gamma^{2}\left[\frac{S(q)}{T \chi_{s}(q)}\right]=\left(\frac{m}{T}\right)^{2}+\left(\frac{c}{T}\right)^{2} q^{2} \text { for } N=\infty .
$$

\section{2. $1 / N$ Expansion}

In this Subsection, we discuss corrections to the $N=$ $\infty$ equations (A10,A13) for the physical case of $N=3$. We utilize the results of the $1 / N$ expansion calculations by Chubukov, Sachdev, and Ye [5] and find that Eqs.(A13,A10) remain quite accurate even for $N=3$.

We turn first to Eq.(A13). While the full q-dependence of $1 / N$ corrections have not been calculated so far, the results for small wavevectors as presented in [5] turn out to be quite sufficient for our purposes. In the asymptotic high temperature (quantum critical) regime, $m / T$ is temperature independent, while $\chi_{s}(0) / \chi_{s}(q)$ and $S(q) / T \chi_{s}(q)$ depend only on the product $q \xi \sim c q / T$. 
With straightforward application of the results of [5], we obtain for small $q$ :

$$
\begin{gathered}
\frac{\chi_{s}(0)}{\chi_{s}(q)}=1+\left(v_{1} \frac{c}{m}\right)^{2} q^{2} \\
\gamma^{2}\left[\frac{S(q)}{T \chi_{s}(q)}\right]=\left(u_{2} \frac{m}{T}\right)^{2}+\left(v_{2} \frac{c}{T}\right)^{2} q^{2}
\end{gathered}
$$

where

$$
\begin{aligned}
& v_{1}=1-0.0042 / N \rightarrow 0.9986, \\
& u_{2}=1+0.1473 / N \rightarrow 1.0491, \\
& v_{2}=1+0.0382 / N \rightarrow 1.0127
\end{aligned}
$$

\section{$(1 / N$ terms are evaluated for $N=3)$.}

The $1 / N$ corrections to the coefficients $v_{1}, v_{2}$, which enter in parenthesis in front of $q^{2}$ in Eqs.(A14,A15), are essentially negligible. Higher terms in the expansion in powers of $q^{2}$ have not been calculated so far. However, once the leading terms have very small corrections, corrections to higher order terms in the expansion in $q^{2}$ should be of the same order of magnitude. The reason for this conclusion is as follows. Eqs.(A14,A15) can be considered as relationshps between the position of the closest to the origin singuliarity for imaginary wavevectors, $q_{\text {sing }}=1 / \xi$, and the expansion in powers of $q^{2}$ near $q=0$. Were the higher order terms large, the position of the singuliarity expressed in terms of the coefficient in front of $q^{2}$, namely, $v_{1}$ or $v_{2}$, would change, which in its turn would lead to large corrections to $v_{1}, v_{2}$. Given the fact that such corrections are in fact small, and ignoring the possibility of accidental cancellations for both Eqs.(A14,A15), we arrive to the conclusion that higher order $q^{2 n}$ terms are small and therefore Eqs.(A14,A15) are applicable for $q a \ll 1$, not only for the much narrower wavector range $q \xi \ll 1$.

It is quite remarkable that the $1 / N$ correction to the coefficient $u_{2}$ is small even when evaluated at $N=3$. Indeed, the ratio $S(\mathbf{Q}) / T \chi(\mathbf{Q})$ reflects the distribution of the spectral weight over frequencies. At the commensurate wavevector, the character of this distribution changes from a delta-function peak at finite $\omega=m$ to a smooth peak centered at $\omega=0$ as one goes from $N=\infty$ to $N=3$, which in general could have led to large $1 / N$ corrections to $u_{2}$. The fact that the rigorously calculated correction (A16) is in fact small shows that already the $N=\infty$ approximation accurately captures the relationship between the spin wave mass and the relative correlation strength at different Matsubara frequencies.

Further, since the above corrections are essentially negligible in the $\mathrm{QC}$ regime where they have been calculated, it iseems reasonable to conjecture that they may not increase drastically as $m / T$ decreases, in which case they should apply to the RC-QC crossover regime as well. The rigorous calculations to check this conjecture are prohibitively complicated and therefore have not been carried out. Instead, in the next Section we verify Eqs.(A14,A15) are in agreement with the numerical data for Heisenberg models for all temperatures studied, and thereby establish that the above conjecture is indeed correct, which greatly simplifies futher considerations.

To summarize, in this Appendix we derived surprisingly simple Eqs.(A14,A15,A16). Negligible (for $v_{1}, v_{2}$ ) or small $\left(\sim 5 \%\right.$ for $\left.u_{2}\right) 1 / N$ corrections to the $N=\infty$ result indicate a well behaved large $N$ expansion, and we expect the above equations to be accurate for the physical $O(3)$ model. The correspondence between the Heisenberg and sigma-models is such that $\chi_{s}(q, \omega)$ for the $\sigma$-model is $\chi(|\mathbf{q}-\mathbf{Q}|, \omega)$ for the Heisenberg model, where $\mathbf{Q}=(\pi, \pi)$. Taking this into account, we rewrite Eqs.(A14,A15) in the form appropriate for the Heisenberg models, Eqs.(12,13), and use them in Section III for comparisons with the numerical data.

\section{APPENDIX B: CORRELATION LENGTH}

In this Appendix, we verify our resluts for $m$ and $c$ by comparing the ratio $c / m$ with the independently calculated correlation length. We calculate the correlation length, defined as the inverse rate of exponential decay of spin correlator at large distances, directly using our new "imaginary wavevector" method [23]. The idea of this calculation is based on an observation that when the pair correlation function decays exponentially up to powerlaw corrections, which is the case for two-dimensional Heisenberg models, the Fourier transform of spin correlator around the ordering wavevector, $\chi\left(\mathbf{Q}+\mathbf{n}_{q} q\right)$, where $\mathbf{n}_{q}$ is a unit vector, should have a singuliarity on the imaginary $q$ axis at $q_{\text {sing }}=i / \xi$ when analytically continued to complex $q$. The position of this singuliarity can be determined through high temperature series expansions using the methods described in [23]. In Fig.7, our results for $\xi$ for the $\mathrm{S}=1 / 2$ Heisenberg model are plotted along with the earlier quantum Monte Carlo calculations by Makivic and Ding [10]; the agreement is excellent at all temperatures.

Now we can verify the equality $\xi=c / m$, which holds by the definition of $m$. In Fig. 8 , we plot our directly calculated $\xi$ by a solid line, the ratio $c / m$ determined solely from Eq.(12) as hollow circles, and the ratio of $c$ and $m$ with both determined seperately from Eq.(13) as solid circles. The agreement between all three sets of data is excellent for both $S=1$ and $S=1 / 2$. This agreement not only indicates that our calculations of $m$ and $c$ are accurate, but also serves as another verification that the $\sigma$-model corectly describes wavevector and frequency dependences of the dynamical susceptibility of the Heisenberg models in the studied temperature range. 


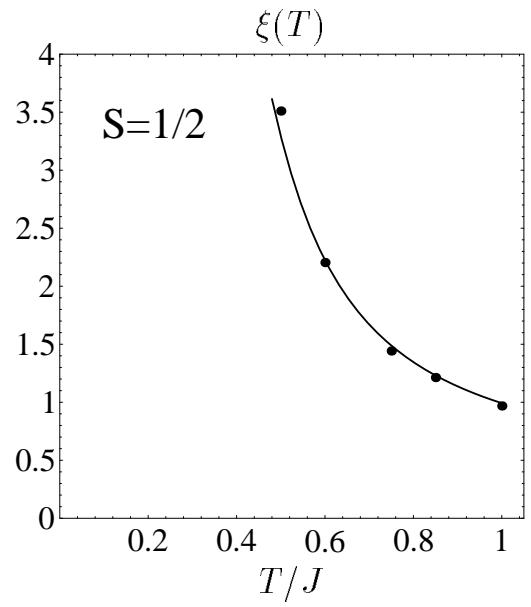

FIG. 7. The correlation length determined using our new method of analytical continuation to imaginary wavevectors (solid line) plotted along with earlier Monte Carlo results or Makivic and Ding [10] (circles).
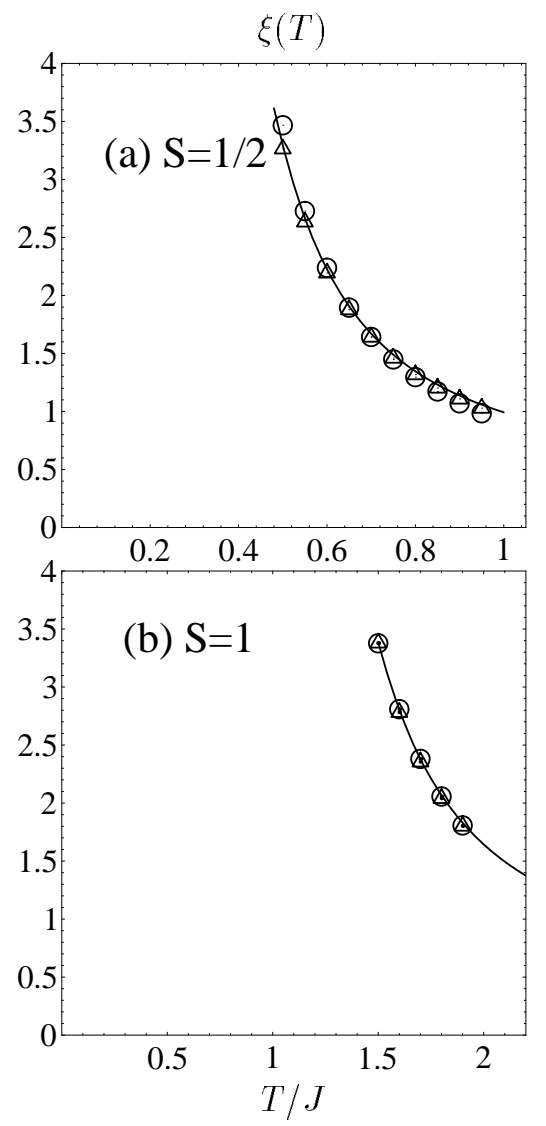

FIG. 8. The correlation length for the $\mathrm{S}=1$ Heisenberg model calculated directly using the analytical continuation to imaginary wavevectors (solid line), and independently using the mapping to the quantum nonlinear $\sigma$-model, from Eq.(12) (triangles) and Eq.(13) (circles). All three sets of data agree with each other for both $S=1 / 2$ and $S=1$. 